\title{
Nonlinear aeroelastic modeling via conformal mapping and vortex method for a flat-plate airfoil in arbitrary motion
}

\author{
Cristina Riso $^{\mathrm{a}}$, Giorgio Riccardi ${ }^{\mathrm{b}, \mathrm{c}}$, Franco Mastroddi ${ }^{*, a}$ \\ ${ }^{a}$ Department of Mechanical and Aerospace Engineering, Sapienza University of Rome \\ via Eudossiana 18, 00184 Rome, Italy. \\ ${ }^{b}$ Department of Industrial and Information Engineering, Second University of Naples \\ via Roma 29, 81031 Aversa (CE), Italy. \\ ${ }^{c}$ CNR-INSEAN, National Research Council of Italy, Maritime Research Center \\ via di Vallerano 139, 00128 Rome, Italy.
}

\begin{abstract}
A nonlinear aerodynamic modeling based on conformal mapping is presented to obtain semi-analytical formulas for the unsteady aerodynamic force and pitching moment on a flat-plate airfoil in arbitrary motion. The aerodynamic model accounts for large amplitudes and non-planar wake and is used to study the aeroelastic behavior of a flat-plate airfoil elastically connected to a support. The fluid is assumed to be inviscid and incompressible, while the flow is assumed to be attached, planar, and potential. Within these hypotheses, conformal mapping and a complex-potential representation of unsteady aerodynamics are used to simplify the theoretical formulation. The vorticity shed at the trailing edge is discretized in desingularized point vortices in order to allow free-wake dynamics. The unsteady aerodynamic model is validated with classical linearized formulations based on the assumption of small disturbances, and with experimental data and theoretical predictions for a largeamplitude pitch-up, hold, pitch-down maneuver. The aeroelastic model is then used to simulate the response of a flat-plate airfoil to sudden starts and body-vortex interactions. Numerical results show that the proposed approach can be an effective tool to model the aeroelastic behavior of an arbitrarily-moving wing section in a time-dependent potential stream of incompressible fluid.
\end{abstract}

Key words: 2D Unsteady Aerodynamics, Aeroelasticity, Vortex Method, Conformal Mapping

\section{Nomenclature}

Latin symbols

$$
\begin{aligned}
\boldsymbol{a}^{(k)} & =\text { wake complex coefficient } \\
\boldsymbol{b}^{\mp} & =\text { wake complex coefficient } \\
\boldsymbol{c}^{\mp} & =\text { wake complex coefficient } \\
\boldsymbol{d} & =\text { wake complex coefficient } \\
D & =\text { drag } \\
f_{x} & =\text { natural frequency of the horizontal linear spring } \\
f_{y} & =\text { natural frequency of the vertical linear spring }
\end{aligned}
$$

${ }^{*}$ Corresponding author. Email: franco.mastroddi@uniroma1.it 


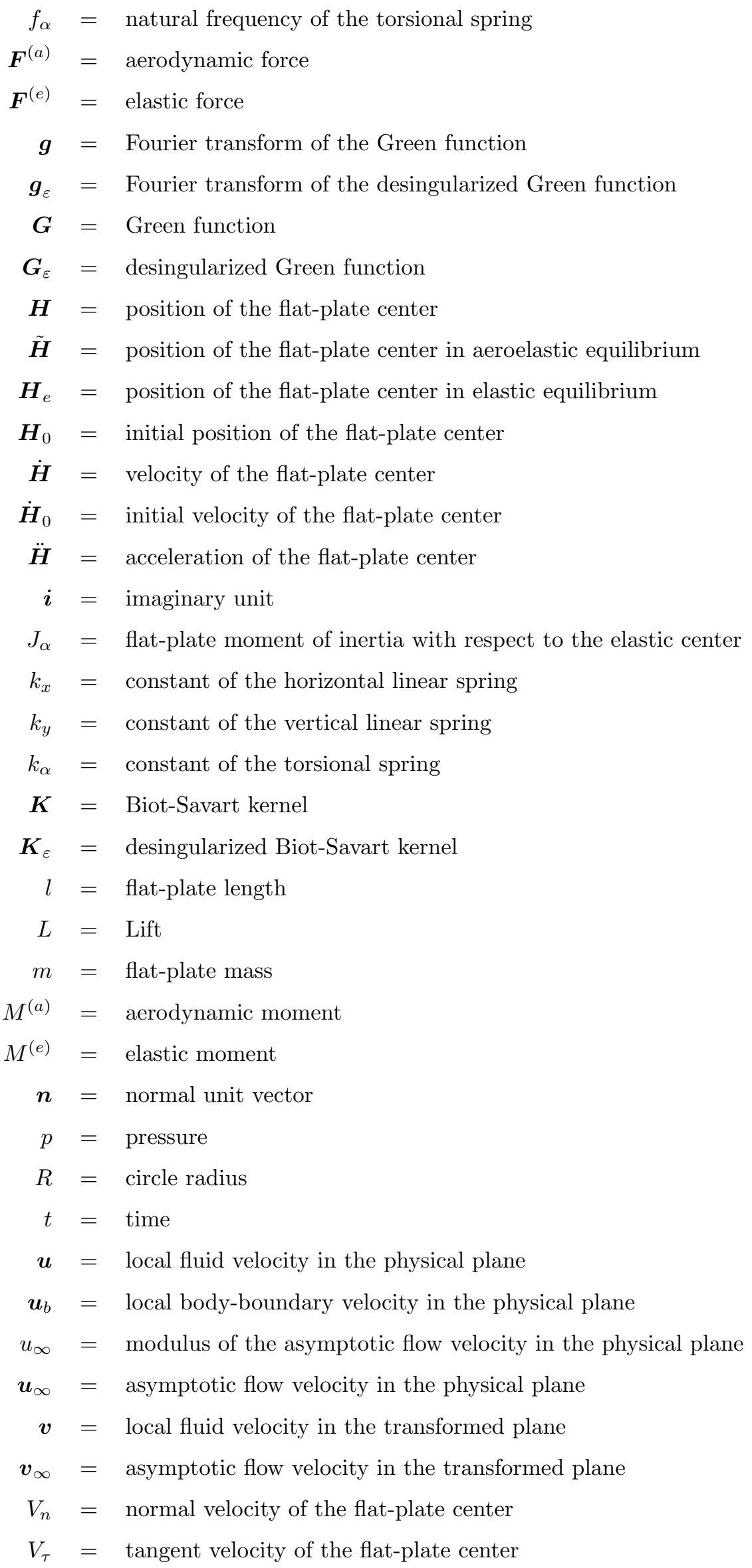




$$
\begin{aligned}
\boldsymbol{w} & =\text { complex potential in the physical plane } \\
\tilde{\boldsymbol{w}} & =\text { complex potential in the transformed plane } \\
\boldsymbol{x} & =\text { position in the physical plane } \\
\boldsymbol{x}_{j} & =\text { position of the } j \text {-th vortex in the physical plane } \\
\boldsymbol{x}_{ \pm} & =\text {position of the plate leading }(-) \text { and trailing }(+) \text { edge in the physical plane }
\end{aligned}
$$

Greek symbols

$$
\begin{aligned}
& \alpha=\text { angle of attack } \\
& \tilde{\alpha}=\text { angle of attack in aeroelastic equilibrium } \\
& \alpha_{e}=\text { angle of attack in elastic equilibrium } \\
& \alpha_{0}=\text { initial angle of attack } \\
& \dot{\alpha}=\text { pitch rate } \\
& \dot{\alpha}_{0}=\text { initial pitch rate } \\
& \ddot{\alpha}=\text { pitch acceleration } \\
& \beta=\text { phase of the asymptotic flow velocity in the physical plane } \\
& \Gamma_{b}=\text { body circulation } \\
& \tilde{\Gamma}_{b}=\text { body circulation in aeroelastic equilibrium } \\
& \Gamma_{b 0}=\text { initial body circulation } \\
& \Gamma_{j}=j \text {-th vortex circulation } \\
& \Gamma^{\star}=\text { nascent vortex circulation } \\
& \delta=\text { parameter to locate the nascent vortex in the transformed plane } \\
& \varepsilon=\text { parameter to desingularize the Biot-Savart kernel } \\
& \boldsymbol{\zeta}=\text { position in the transformed plane } \\
& \boldsymbol{\zeta}_{j}=\text { position of the } j \text {-th vortex in the transformed plane } \\
& \boldsymbol{\zeta}_{ \pm}=\text {position of the plate leading }(-) \text { and trailing }(+) \text { edge in the transformed plane } \\
& \zeta^{\star}=\text { initial position of the nascent vortex in the transformed plane } \\
& \mu=\text { added-to-airfoil moment of inertia ratio } \\
& \rho=\text { fluid density } \\
& \sigma=\text { added-to-airfoil mass ratio } \\
& \tau=\text { tangent unit vector } \\
& \varphi=\text { velocity potential } \\
& \boldsymbol{\Phi}=\text { Schwarz function of the body boundary } \\
& \chi=\text { point on the unit circle } \\
& \psi=\text { stream function } \\
& \Omega_{b}=\text { body cross-section }
\end{aligned}
$$


Operators

$$
\begin{aligned}
\overline{(\cdot)} & =\text { complex conjugate } \\
(\cdot)_{n} & =\text { normal component } \\
(\cdot)_{\tau} & =\text { tangent component } \\
(\cdot)_{x} & =\text { real part } \\
(\cdot)_{y} & =\text { imaginary part } \\
d(\cdot) & =\text { differential } \\
\partial_{t}(\cdot) & =\text { time derivative } \\
\partial_{\boldsymbol{x}}(\cdot) & =\text { complex derivative in the physical plane } \\
\partial_{\boldsymbol{\zeta}}(\cdot) & =\text { complex derivative in the transformed plane }
\end{aligned}
$$

\section{Introduction}

Aeroelastic design is typically tackled by means of linearized approaches and well-established associated computational tools. The linearization process typically includes the assumptions of linearized body kinematics and prescribed wake geometry. However, these simplifications may be not reasonable, for example, when analyzing aircraft configurations undergoing large-amplitude static and dynamic deflections. On the other hand, high-fidelity nonlinear aeroelastic models are computationally demanding, thus not suitable whenever multiple simulations have to be performed. Therefore, there is a need of simplified models, capable of accounting for relevant aerodynamic and structural nonlinearities with moderate computational burden, to be used in sensitivity analysis, optimization, and control.

In a linear framework, typical-section aeroelastic models were historically the first example of analytical tools used for prediction and design. The possibility to derive closed-form solutions for the unsteady aerodynamic loads under the assumption of small disturbances has made such models an important source of information on unsteady airfoil behavior and fixed-wing flutter. Wagner (Ref. [1]) investigated the lift response to a step of angle of attack for a flat-plate airfoil in incompressible potential flow. Küssner (Ref. [2]) addressed the similar problem for the case of a vertical gust. Theodorsen (Refs. [3, 4]) presented the lift and pitching moment on a thin airfoil with a flap undergoing small harmonic oscillations in plunge and pitch, namely in a frequency-domain description. His major contribution was to analytically quantify the circulatory loads due to a continuous planar vortex sheet through a unique function of the reduced frequency (the so-called Theodorsen function). Using the obtained model for the lift and pitching moment, he also carried out a detailed investigation on the mechanism of flutter (Refs. $[3,5]$ ), aimed to point out influence of typical-section properties on stability margin. Garrick (Ref. [6]) demonstrated that the Wagner and Küssner functions are related to the Theodorsen function by frequency-domain relationships. Isaacs (Ref. [7]) evaluated the lift on a thin airfoil in a time-dependent free stream. Similarly, Greenberg (Ref. [8]) derived an extension of the Theodorsen theory including an unsteady incoming flow, and compared his solution with the Isaacs theory.

Linear unsteady aerodynamic models for flat-plate airfoils have been later extended in the Laplace domain and recast in state-space form (Refs. $[9,10]$ ) in order to be used in the control-theory framework. Finite-state aerodynamic models were first obtained via numerical fitting of either the Wagner function in the time domain 
or the Theodorsen function in the frequency domain (Refs. $[11,12,13]$ ). More recently, aerodynamic models in state-space form were also deduced from the governing equations of the aerodynamic problem (Refs. [14, 15]). According to this latter approach, the aerodynamic states have the physical interpretation of inflow distributions.

Due to the growing interest in flapping wings and unsteady aerodynamics of micro aerial vehicles (MAVs), recent research has focused on improving two-dimensional unsteady airfoil theory for incompressible potential flows in order to account for large amplitudes and non-planar wakes. Ramesh et al. (Ref. [16]) proposed a largeangle unsteady thin airfoil theory to predict the lift coefficient using a chordwise vorticity distribution to satisfy the no-penetration unsteady boundary condition and a time-stepping approach to compute the wake downwash. The model was later refined including leading-edge vortex shedding (Ref. [17]). Xia and Mohseni (Ref. [18]) explored the influence of leading-edge and trailing-edge vortex shedding from a pitching flat plate using a point vortex method (Refs. [19, 20]) based on a complex-potential representation for the flow (Refs. [21, 22]) and conformal mapping (Ref. [23]). The complex potential was derived by applying the Milne-Thomson circle theorem (Ref. [22]) in a non-inertial body-fixed frame of reference and using a multi-vortices model to account for the wake vorticity. The aerodynamic force was analytically evaluated via the unsteady Blasius theorem (Ref. [22]). Wang and Eldredge (Ref. [24]) proposed a low-order aerodynamic model for a flat plate undergoing large-amplitude maneuvers using finite sets of point vortices of variable strengths to approximate the vorticity shed from the section edges. A time-dependent conformal map was adopted to obtain the complex potential by imposing the unsteady boundary condition on the plate and adding the vortex-induced contributions. The aerodynamic force and pitching moment were deduced from the linear and angular impulses and analytically evaluated using the residue theorem (Ref. [23]). Yan et al. (Ref. [25]) developed an unsteady potential flow model for a flat-plate airfoil including nonlinear kinematics and non-planar wake. Following the Theodorsen method (Refs. [4, 3]), a velocity potential evaluated via a stationary conformal map was used to obtain the non-circulatory and circulatory loads.

Besides accounting for nonlinearities, another challenge of recent interest in theoretical modeling of unsteady aerodynamics is including chordwise flexibility in thin airfoil models, with the aim to exploit combinations of rigid-body and elastic motions for propulsion. Unfortunately, most of the modeling approaches suitable to address large amplitudes and non-planar wakes are limited to the case of rigid sections, which precludes inclusion of chordwise flexibility in a nonlinear framework. Indeed, the no-penetration condition is usually imposed in body-fixed frames of reference, in which rigid bodies are at rest, in order to use classical theoretical tools for aerodynamic modeling (e.g., Ref. [18]). This procedure also enables to obtain the aerodynamic loads via the unsteady Blasius theorem, which holds for stationary bodies or, in the case of rigid-body motions, in bodyfixed frames of reference. Although a more general approach is followed in Ref. [24], nevertheless the evolution equation there presented for the variable-strength vortex singularities only applies to flat plates. On the other hand, Walker and Patil (Ref. [26]) derived an unsteady aerodynamic model for flexible thin airfoils, but still assuming small disturbances.

In this framework, the present work proposes a general formulation to predict the unsteady aerodynamic force and pitching moment on moving airfoils in incompressible potential flow. The aerodynamic loads are deduced by locally imposing the no-penetration unsteady boundary condition on the body wall, with no theoretical 
restrictions on the section shape and motion. Therefore, the present formulation can be applied to different and also deformable airfoils. The modeling approach is based on a complex-potential representation of the flow (Refs. [21, 22]) and exploit features of the theory of analytic functions (Refs. [23, 27, 28]). In particular, the Schwarz function of the body boundary (Ref. [27]) is used to recast the aerodynamic loads on moving boundaries in complex forms suitable to apply the residue theorem.

In order to assess the fidelity of the modeling approach, the present study focuses on the simple case of a flat-plate airfoil undergoing plunge, surge, and pitch motions, with the aim to address a flexible thin airfoil in future research. A time-dependent conformal map equivalent to the one in Ref. [24] is used to identify the complex potential by locally imposing the unsteady boundary condition on the body wall. A sequence of desingularized point vortices shed from the trailing edge and moving with their local velocities completes the unsteady aerodynamic model. The aerodynamic force and pitching moment on a flat plate are obtained by specializing the general formulation and evaluated via the residue theorem (Ref. [23]). Semi-analytical formulas for the aerodynamic loads including nonlinear effects due to large-amplitude motions and free-wake dynamics are presented and validated against existing theoretical models and experiments. The aerodynamic model is then used to study the aeroelastic behavior of a flat-plate airfoil elastically connected to a rigid support.

The paper is organized as follows. General formulas for the unsteady aerodynamic force and pitching moment on airfoil sections in arbitrary motion are deduced in Sec. 2. The formulation is specialized to a flat-plate airfoil in Sec. 3. An aeroelastic model based on the proposed aerodynamic model is presented in Sec. 4. Numerical results are discussed in Sec. 5. The unsteady aerodynamic model is first validated with classical linearized formulations for the case of small disturbances, and with the experimental data and theoretical predictions by Ramesh et al. (Ref. [16]) for a large-amplitude pitch-up, hold, pitch-down maneuver. The aeroelastic model is then used to perform free-wake simulations of the response to a sudden start and to the interaction with a passing isolated vortex. Finally, concluding remarks are given in Sec. 6 .

\section{Aerodynamic force and moment on moving airfoils}

In this section a general formulation for the unsteady aerodynamic force and pitching moment on moving airfoils in incompressible potential flow is presented. The aerodynamic loads are deduced by imposing that the normal components of the body and fluid velocities be equal at the body wall, with no theoretical restrictions on the section shape and motion. The aim is to derive general formulas applicable to different common airfoil sections undergoing arbitrary motion, also including chordwise deformation (like flexible thin airfoils).

In order to achieve such generality, the no-penetration condition must be locally imposed on a moving body boundary rather than in body-fixed frame of reference, as usually done for rigid sections. This implies some difficulties when recasting the aerodynamic loads into complex forms suitable to apply the residue theorem (Ref. [23]). Indeed, unsteady boundary conditions typically introduce a dependency on the conjugate position in the integrands that give the aerodynamic force and moment. As a consequence, such integrands are not anymore analytic because of the body motion, so that the aerodynamic loads could not be evaluated via the residue theorem unless the conjugate position is rewritten as an analytic function. This problem is here 
addressed by exploiting the special properties of the Schwarz function (Ref. [27]) of the body boundary, as it will be explained below.

The flow is planar, so representing an arbitrary section of a wing along the span. The plane of the flow is identified with the complex one. A complex quantity is written with a bold character (e.g., $\boldsymbol{i}$ is the imaginary unit), and the conjugate is denoted by an overline. The airfoil section is assumed as a simply-connected bounded domain $\Omega_{b}$, having the finite-length, piecewise smooth boundary $\partial \Omega_{b}$. This closed curve is counterclockwise oriented with a tangent unit vector $\boldsymbol{\tau}$, so that the outward normal unit vector is $\boldsymbol{n}=-\boldsymbol{i} \boldsymbol{\tau}$. The domain $\Omega_{b}$ (and then its boundary $\partial \Omega_{b}$ ) is time-dependent: its position and orientation in the plane of the flow vary smoothly in time while the body moves, as well as its shape. Any point of the section boundary moves with a known velocity $\boldsymbol{u}_{b}$, which depends smoothly on the position $\boldsymbol{x}$ and time $t$. The flow is assumed to be irrotational and a complex potential $\boldsymbol{w}=\varphi+\boldsymbol{i} \psi$ is introduced, where $\varphi$ and $\psi$ are the velocity potential and the stream function, respectively. The complex potential is an analytic function of the position, and a smooth function of time. The complex derivative $\partial_{\boldsymbol{x}} \boldsymbol{w}$ gives the local conjugate flow velocity $\overline{\boldsymbol{u}}=u-\boldsymbol{i} v$ (Refs. [23, 21]).

The present formulation for the aerodynamic force and moment is based on the use of the Schwarz function $\mathbf{\Phi}$ of a curve (Ref. [27]), specifically of the curve $\partial \Omega_{b}$ in this case, in order to rewrite complex integrands depending on the conjugate position as analytic functions. Indeed, it is well known that the conjugate position $\overline{\boldsymbol{x}}$ is not an analytic function of $\boldsymbol{x}$. However, it is a smooth function of $\boldsymbol{x}$ at any point that belongs to the finite-length, piecewise smooth curve $\partial \Omega_{b}$. The Schwarz function $\boldsymbol{\Phi}$ of $\partial \Omega_{b}$ is then defined as the analytic continuation of the function $\overline{\boldsymbol{x}}=\overline{\boldsymbol{x}}(\boldsymbol{x})$ mentioned above for $\boldsymbol{x}$ belonging to a suitable neighborhood of $\partial \Omega_{b}$. The size of the neighborhood depends on the positions of the singular points ${ }^{1}$ of $\boldsymbol{\Phi}$. Note that $\boldsymbol{\Phi}(\boldsymbol{x})$ is different from the conjugate position $\overline{\boldsymbol{x}}$ for $\boldsymbol{x} \notin \partial \Omega_{b}$, whereas it is equal to $\overline{\boldsymbol{x}}$ for $\boldsymbol{x} \in \partial \Omega_{b}$. This allows to replace $\overline{\boldsymbol{x}}$ by $\boldsymbol{\Phi}$ on the body boundary, and to evaluate the conjugate curve element $d \overline{\boldsymbol{x}}$ as $d \boldsymbol{x} \partial_{\boldsymbol{x}} \boldsymbol{\Phi}$. These properties of the Schwarz function will be used in the derivation that follows to recast the aerodynamic loads in complex forms suitable to apply the residue theorem.

The unsteady aerodynamic force $\boldsymbol{F}^{(a)}$ (per unit span length) on a moving airfoil $\Omega_{b}$ is derived below. Since the fluid is assumed to be inviscid, the force is only due to pressure. Using the Bernoulli theorem, one has:

$$
\boldsymbol{F}^{(a)}=-\boldsymbol{i} \rho \int_{\partial \Omega_{b}} d \boldsymbol{x}\left(\partial_{t} \varphi+\frac{|\boldsymbol{u}|^{2}}{2}\right),
$$

where $\rho$ is the (uniform and constant) fluid density. The unsteady term, i.e. the first contribution in Eq. (1),

\footnotetext{
${ }^{1}$ The cases of a circle and an ellipse are discussed below. The conjugate position on the circle of radius $R$ and center at the origin is written in terms of the angle $\theta \in[0,2 \pi)$ as $\overline{\boldsymbol{x}}=R \exp (-\boldsymbol{i} \theta)$, which yields the Schwarz function $\boldsymbol{\Phi}(\boldsymbol{x})=R^{2} / \boldsymbol{x}$ with a simple pole at $\boldsymbol{x}=0$. The position $\boldsymbol{x}$ and its conjugate $\overline{\boldsymbol{x}}$ on the ellipse of semi-major axis $a$ (along $x$ ), semi-minor axis $b$ (along $y$ ), and half-focal separation $c$ are written in terms of the angle $\theta \in[0,2 \pi)$ as:

$$
\boldsymbol{x}=[(a+b) \exp (\boldsymbol{i} \theta)+(a-b) \exp (-\boldsymbol{i} \theta)] / 2, \quad \overline{\boldsymbol{x}}=[(a+b) \exp (-\boldsymbol{i} \theta)+(a-b) \exp (\boldsymbol{i} \theta)] / 2 .
$$

Choosing the principal branch of the square root, one has from the first relation $\exp (i \theta)=[\boldsymbol{x}+\sqrt{(\boldsymbol{x}-c)(\boldsymbol{x}+c)}] /(a+b)$. Once this is substituted into $\overline{\boldsymbol{x}}$, the Schwarz function turns out to be:

$$
\boldsymbol{\Phi}(\boldsymbol{x})=\frac{\left(a^{2}+b^{2}\right) \boldsymbol{x}-a b \sqrt{\boldsymbol{x}^{2}-c^{2}}}{c^{2}},
$$

with a branch cut along the focal axis across which the phase exhibits a jump of $+\pi$.
} 
is rewritten as a complex integral using the relations $\partial_{t} \varphi=\left(\partial_{t} \boldsymbol{w}+\overline{\partial_{t} \boldsymbol{w}}\right) / 2$ and $d \overline{\boldsymbol{x}}=d \boldsymbol{x} \partial_{\boldsymbol{x}} \boldsymbol{\Phi}$ :

$$
\int_{\partial \Omega_{b}} d \boldsymbol{x} \partial_{t} \varphi=\frac{1}{2}\left(\int_{\partial \Omega_{b}} d \boldsymbol{x} \partial_{t} \boldsymbol{w}+\overline{\int_{\partial \Omega_{b}} d \boldsymbol{x} \partial_{\boldsymbol{x}} \boldsymbol{\Phi} \partial_{t} \boldsymbol{w}}\right) .
$$

In order to put the steady term, i.e. the second contribution in Eq. (1), in complex form, the differential of the stream function along $\partial \Omega_{b}$ is written using the no-penetration unsteady boundary condition as $d \psi=d s \boldsymbol{u} \cdot \boldsymbol{n}=$ $d s \boldsymbol{u}_{b} \cdot \boldsymbol{n}=\boldsymbol{i}\left(\boldsymbol{u}_{b} d \overline{\boldsymbol{x}}-\overline{\boldsymbol{u}}_{b} d \boldsymbol{x}\right) / 2$. Therefore, one obtains on $\partial \Omega_{b}$ :

$$
d \boldsymbol{w}=d \varphi+\frac{1}{2}\left(\overline{\boldsymbol{u}}_{b} d \boldsymbol{x}-\boldsymbol{u}_{b} d \overline{\boldsymbol{x}}\right) .
$$

Using this relation twice, the steady term becomes:

$$
\begin{aligned}
& \int_{\partial \Omega_{b}} d \boldsymbol{x} \frac{|\boldsymbol{u}|^{2}}{2}=\frac{1}{2} \int_{\partial \Omega_{b}} d \boldsymbol{w} \overline{\partial_{\boldsymbol{x}} \boldsymbol{w}} \\
& =\frac{1}{2} \overline{\int_{\partial \Omega_{b}} d \varphi \partial_{\boldsymbol{x}} \boldsymbol{w}}+\frac{1}{4} \int_{\partial \Omega_{b}}\left(\overline{\boldsymbol{u}}_{b} d \boldsymbol{x}-\boldsymbol{u}_{b} d \overline{\boldsymbol{x}}\right) \overline{\partial_{\boldsymbol{x}} \boldsymbol{w}} \\
& =\frac{1}{2} \overline{\int_{\partial \Omega_{b}}\left[d \boldsymbol{w}-\frac{1}{2}\left(\overline{\boldsymbol{u}}_{b} d \boldsymbol{x}-\boldsymbol{u}_{b} d \overline{\boldsymbol{x}}\right)\right] \partial_{\boldsymbol{x}} \boldsymbol{w}}+\frac{1}{4} \int_{\partial \Omega_{b}}\left(\overline{\boldsymbol{u}}_{b} d \boldsymbol{x}-\boldsymbol{u}_{b} d \overline{\boldsymbol{x}}\right) \overline{\partial_{\boldsymbol{x}} \boldsymbol{w}} \\
& =\frac{1}{2}\left(\overline{\int_{\partial \Omega_{b}} d \boldsymbol{x}\left(\partial_{\boldsymbol{x}} \boldsymbol{w}\right)^{2}}+\overline{\int_{\partial \Omega_{b}} d \boldsymbol{x} \partial_{\boldsymbol{x}} \boldsymbol{\Phi} \boldsymbol{u}_{b} \partial_{\boldsymbol{x}} \boldsymbol{w}}-\overline{\int_{\partial \Omega_{b}} d \boldsymbol{x} \overline{\boldsymbol{u}}_{b} \partial_{\boldsymbol{x}} \boldsymbol{w}}\right) \text {. }
\end{aligned}
$$

Substituting Eqs. (2) and (4) into Eq. (1), the aerodynamic force is rewritten in the general complex form:

$$
\boldsymbol{F}^{(a)}=-\frac{\boldsymbol{i} \rho}{2}\left[\int_{\partial \Omega_{b}} d \boldsymbol{x} \partial_{t} \boldsymbol{w}+\overline{\int_{\partial \Omega_{b}} d \boldsymbol{x} \partial_{\boldsymbol{x}} \boldsymbol{\Phi}\left(\partial_{t} \boldsymbol{w}+\boldsymbol{u}_{b} \partial_{\boldsymbol{x}} \boldsymbol{w}\right)}+\overline{\int_{\partial \Omega_{b}} d \boldsymbol{x} \partial_{\boldsymbol{x}} \boldsymbol{w}\left(\partial_{\boldsymbol{x}} \boldsymbol{w}-\overline{\boldsymbol{u}}_{b}\right)}\right]
$$

Note that the first two integrals in $\boldsymbol{F}^{(a)}$ become identical if the body does not move $\left(\boldsymbol{u}_{b} \equiv 0\right)$. Indeed, the stream function is constant in space along the body boundary in this situation, namely one has $\psi=\psi_{0}(t)$ on $\partial \Omega_{b}$. As a consequence, the integrals of the imaginary part of $\partial_{t} \boldsymbol{w}$ (given by $\boldsymbol{i} \dot{\psi}_{0}$ ) vanish in Eq. (5), whereas those of the real parts are equal. Therefore, Eq. (5) reduces to the unsteady Blasius theorem (Refs. [18, 22]):

$$
\boldsymbol{F}^{(a)}=-\boldsymbol{i} \rho \int_{\partial \Omega_{b}} d \boldsymbol{x} \partial_{t} \boldsymbol{w}-\frac{\boldsymbol{i} \rho \overline{2}}{2} \overline{\int_{\partial \Omega_{b}} d \boldsymbol{x}\left(\partial_{\boldsymbol{x}} \boldsymbol{w}\right)^{2}}
$$

which accounts for flow unsteadiness but not for body motions. Moreover, Eq. (5) reduces to the classical Blasius theorem (Refs. $[21,22])$ in the case of stationary bodies in steady flows $\left(\partial_{t} \boldsymbol{w} \equiv 0\right)$.

The component of the aerodynamic moment (per unit span length) normal to the plane of the motion (namely along the $z$-axis), denoted by $M^{(a)}$, is written with respect to the pole $\boldsymbol{q}$ as:

$$
M^{(a)}(\boldsymbol{q})=\int_{\partial \Omega_{b}} d s(\boldsymbol{x}-\boldsymbol{q}) \times\left.(-p \boldsymbol{n})\right|_{z}=M^{(a)}(0)-\boldsymbol{q} \times\left.\boldsymbol{F}^{(a)}\right|_{z}
$$

where $M^{(a)}(0)$ is the moment with respect to the origin $(\boldsymbol{q}=0)$. The complex form of the latter is obtained by rewriting the $z$-component of $\boldsymbol{x} \times \boldsymbol{n}$ as $-\operatorname{Re}(\boldsymbol{\tau} \boldsymbol{\Phi})$ :

$$
M^{(a)}(0)=-\operatorname{Re}\left[\int_{\partial \Omega_{b}} d \boldsymbol{\Phi}(-p)\right]=-\rho \operatorname{Re}\left(\int_{\partial \Omega_{b}} d \boldsymbol{x} \boldsymbol{\Phi} \partial_{t} \varphi+\frac{1}{2} \int_{\partial \Omega_{b}} d \boldsymbol{\Phi} \overline{\partial_{\boldsymbol{x}} \boldsymbol{w}} \partial_{\boldsymbol{x}} \boldsymbol{w}\right)
$$

Note that the arbitrary function of the time given by $\partial_{t} \varphi+|\boldsymbol{u}|^{2} / 2+p / \rho$ in the Bernoulli theorem does not play any role since the integral of $\boldsymbol{\Phi}$ on $\partial \Omega_{b}$ is imaginary (Ref. [27]). The unsteady and steady terms in Eq. (7) are 
handled as for the force, so that after some manipulation one obtains the complex form:

$$
M^{(a)}(0)=-\frac{\rho}{2} \operatorname{Re}\left[\int_{\partial \Omega_{b}} d \boldsymbol{x} \boldsymbol{\Phi} \partial_{t} \boldsymbol{w}+\int_{\partial \Omega_{b}} d \boldsymbol{x} \boldsymbol{x} \partial_{\boldsymbol{x}} \boldsymbol{\Phi}\left(\partial_{t} \boldsymbol{w}+\boldsymbol{u}_{b} \partial_{\boldsymbol{x}} \boldsymbol{w}\right)+\int_{\partial \Omega_{b}} d \boldsymbol{x} \boldsymbol{x} \partial_{\boldsymbol{x}} \boldsymbol{w}\left(\partial_{\boldsymbol{x}} \boldsymbol{w}-\overline{\boldsymbol{u}}_{b}\right)\right] .
$$

This reduces to the classical Blasius theorem (Refs. [21, 22]) in the case of stationary bodies in steady flows.

\section{Aerodynamic model of a flat-plate airfoil}

In this section the present formulation is specialized to develop the unsteady aerodynamic model of a flat-plate airfoil undergoing plunge, surge, and pitch. The complex potential of the flow is evaluated by locally imposing the no-penetration unsteady boundary condition and using a conformal map to transform the physical plane ( $\boldsymbol{x}$-plane) onto an auxiliary plane ( $\boldsymbol{\zeta}$-plane) in which the flat plate becomes a circle. As in Ref. [24], a time-dependent conformal map that accounts for the body motion is adopted in place of the classical stationary Joukowski map, which is used in linearized formulations or whenever the aerodynamic model is developed in a body-fixed frame of reference (for instance, Refs. [3, 18, 26]). The wake shed from the trailing edge is modeled using a vortex method, which leads to a semi-analytical model requiring a numerical integration of wake dynamics. A new vortex (the so-called nascent vortex) is inserted into the flow field at each time step. The nascent-vortex initial position is prescribed, whereas its circulation is evaluated by enforcing the flow velocity to behave smoothly in a neighborhood of the trailing edge. This procedure is known as the fixed-position method (Ref. [20]). Once shed, the vortices move with the local velocities based on a desingularized Biot-Savart kernel (Refs. $[29,30])$ in order to implement free-wake dynamics. The aerodynamic force and moment are finally evaluated using the residue theorem.

\subsection{Conformal maps}

The flat plate of length $l$, center at the point $\boldsymbol{H}(t)=H_{x}(t)+\boldsymbol{i} H_{y}(t)$ and with a clockwise angle of attack $\alpha(t)$ with respect to the $x$-axis is mapped onto a fixed circle having center at the origin and radius $R$. Introduced the time-dependent point on the unit circle $\boldsymbol{\chi}=\exp (\boldsymbol{i} \alpha)$, the conformal map and its inverse are given by:

$$
\begin{aligned}
\boldsymbol{\zeta} & =2 \chi \frac{R}{l}\left[(\boldsymbol{x}-\boldsymbol{H})+\sqrt{\left(\boldsymbol{x}-\boldsymbol{x}_{-}\right)\left(\boldsymbol{x}-\boldsymbol{x}_{+}\right)}\right] \\
\boldsymbol{x} & =\frac{l \bar{\chi}}{4 R} \boldsymbol{\zeta}+\boldsymbol{H}+\frac{l R \bar{\chi}}{4} \frac{1}{\boldsymbol{\zeta}} .
\end{aligned}
$$

The dependency on the instantaneous plate-center position $\boldsymbol{H}$ includes plunge and surge motions, whereas the dependency on the instantaneous angle of attack accounts for pitch. The time-dependent points $\boldsymbol{x}_{ \pm}:=\boldsymbol{H} \pm l \overline{\boldsymbol{\chi}} / 2$ are the trailing (upper sign) and leading (lower sign) edges, which correspond to the points $\boldsymbol{\zeta}_{ \pm}:= \pm R$ on the circle. The intrinsic reference system $\boldsymbol{\tau}=-\bar{\chi}$ (tangent unit vector), $\boldsymbol{n}=\boldsymbol{i} \overline{\boldsymbol{\chi}}$ (normal unit vector) is adopted, in which the plate-center position and velocity have the following components:

$$
H_{\tau}=-\operatorname{Re}(\boldsymbol{\chi} \boldsymbol{H}), \quad H_{n}=\operatorname{Im}(\boldsymbol{\chi} \boldsymbol{H}), \quad V_{\tau}=-\operatorname{Re}(\boldsymbol{\chi} \dot{\boldsymbol{H}}), \quad V_{n}=\operatorname{Im}(\boldsymbol{\chi} \dot{\boldsymbol{H}}) .
$$

The velocity of the plate boundary is written as:

$$
\boldsymbol{u}_{b}=\dot{\boldsymbol{H}}-\boldsymbol{i} \dot{\alpha}(\boldsymbol{x}-\boldsymbol{H})=\dot{\boldsymbol{H}}-\boldsymbol{i} \bar{\chi} \frac{\dot{\alpha} l}{4}\left(\frac{\boldsymbol{\zeta}}{R}+\frac{R}{\boldsymbol{\zeta}}\right) .
$$


Finally, the asymptotic velocity in the $\boldsymbol{\zeta}$-plane, denoted by $\boldsymbol{v}_{\infty}$, is different from the physical one $\boldsymbol{u}_{\infty}=$ $u_{\infty} \exp (\boldsymbol{i} \beta)$ as the maps in Eq. (9) do not reduce to identities far from the plate (and from the circle). The relation between the asymptotic velocities is:

$$
\boldsymbol{v}_{\infty}=\frac{l \chi}{4 R} \boldsymbol{u}_{\infty}=\frac{l u_{\infty}}{4 R} e^{i(\alpha+\beta)}
$$

\subsection{Complex potential}

The complex potential $\boldsymbol{w}$ in the $\boldsymbol{x}$-plane is obtained from the one $\tilde{\boldsymbol{w}}$ in the $\boldsymbol{\zeta}$-plane through the map $\boldsymbol{\zeta}=\boldsymbol{\zeta}(\boldsymbol{x} ; t)$, namely $\boldsymbol{w}(\boldsymbol{x} ; t)=\tilde{\boldsymbol{w}}[\boldsymbol{\zeta}(\boldsymbol{x} ; t) ; t]$. Hence, its time and space derivatives are evaluated as $\partial_{t} \boldsymbol{w}=$ $\partial_{t} \tilde{\boldsymbol{w}}+\partial_{\boldsymbol{\zeta}} \tilde{\boldsymbol{w}} \partial_{t} \boldsymbol{\zeta}$ and $\partial_{\boldsymbol{x}} \boldsymbol{w}=\partial_{\boldsymbol{\zeta}} \tilde{\boldsymbol{w}} \partial_{\boldsymbol{x}} \boldsymbol{\zeta}$. The complex potential $\tilde{\boldsymbol{w}}$ is the sum of the contributions due to the free stream $\left(\tilde{\boldsymbol{w}}_{\infty}\right)$, plate dynamics $\left(\tilde{\boldsymbol{w}}_{d}\right)$, circulation around the body $\left(\tilde{\boldsymbol{w}}_{c}\right)$, and wake $\left(\tilde{\boldsymbol{w}}_{w}\right)$.

The normal velocity of the plate boundary is evaluated from Eq. (11) by taking $\boldsymbol{\zeta}=R \exp (\boldsymbol{i} \theta)$ as $u_{b n}=$ $\boldsymbol{u}_{b} \cdot \boldsymbol{n}=\operatorname{Re}\left(\overline{\boldsymbol{u}}_{b} \boldsymbol{n}\right)=V_{n}-(\dot{\alpha} l / 2) \cos \theta$. In order to satisfy the unsteady boundary condition on the body wall, the complex potential of the non-circulatory flow is then assumed of the form:

$$
\tilde{\boldsymbol{w}}_{\infty}+\tilde{\boldsymbol{w}}_{d}=\overline{\boldsymbol{v}}_{\infty} \boldsymbol{\zeta}+\frac{A_{r}+\boldsymbol{i} A_{i}}{\boldsymbol{\zeta}}+\frac{B_{r}+\boldsymbol{i} B_{i}}{\boldsymbol{\zeta}^{2}}
$$

so that the corresponding normal velocity on the plate boundary $u_{n}=\boldsymbol{u} \cdot \boldsymbol{n}$ is given by:

$$
\begin{aligned}
u_{n} & =\operatorname{Re}\left[\partial_{\boldsymbol{x}}\left(\boldsymbol{w}_{\infty}+\boldsymbol{w}_{d}\right) \boldsymbol{n}\right] \\
& =\frac{2 R}{l} \frac{1}{\sin \theta}\left[-\frac{2 B_{r}}{R^{3}}+\left(v_{\infty r}-\frac{A_{r}}{R^{2}}\right) \cos \theta+\left(v_{\infty i}-\frac{A_{i}}{R^{2}}\right) \sin \theta-\frac{4 B_{i}}{R^{3}} \sin \theta \cos \theta+\frac{4 B_{r}}{R^{3}} \sin ^{2} \theta\right] .
\end{aligned}
$$

Imposing $u_{n}=u_{b n}$ gives:

$$
\tilde{\boldsymbol{w}}_{\infty}(\boldsymbol{\zeta} ; t)=\overline{\boldsymbol{v}}_{\infty}(t) \boldsymbol{\zeta}+\boldsymbol{v}_{\infty}(t) \frac{R^{2}}{\boldsymbol{\zeta}}, \quad \tilde{\boldsymbol{w}}_{d}(\boldsymbol{\zeta} ; t)=-\boldsymbol{i} \frac{l V_{n}(t)}{2} \frac{R}{\boldsymbol{\zeta}}+\boldsymbol{i} \frac{l^{2} \dot{\alpha}(t)}{16} \frac{R^{2}}{\boldsymbol{\zeta}^{2}}
$$

Note that the complex-potential $\tilde{\boldsymbol{w}}_{d}$ is equivalent to the one derived in Ref. [24].

The overall complex potential is obtained by adding the circulatory terms $\left(\tilde{\boldsymbol{w}}_{c}+\tilde{\boldsymbol{w}}_{w}\right)$. These account for the circulation around the body $\left(\Gamma_{b}\right)$ and for the wake, the latter discretized in vortices. The well-known complex potentials (Ref. [22]) are listed below:

$$
\tilde{\boldsymbol{w}}_{c}(\boldsymbol{\zeta} ; t)=\frac{\Gamma_{b}(t)}{2 \pi \boldsymbol{i}} \log \boldsymbol{\zeta}, \quad \tilde{\boldsymbol{w}}_{w}(\boldsymbol{\zeta} ; t)=\frac{1}{2 \pi \boldsymbol{i}} \sum_{j=1}^{n} \Gamma_{j}\left\{\log \left[\boldsymbol{\zeta}-\boldsymbol{\zeta}_{j}(t)\right]-\log \left[\boldsymbol{\zeta}-R^{2} / \overline{\boldsymbol{\zeta}}_{j}(t)\right]+\log \boldsymbol{\zeta}\right\}
$$

where $\Gamma_{j}$ and $\boldsymbol{\zeta}_{j}=\boldsymbol{\zeta}_{j}(t)$ are the constant circulation and time-dependent position in the $\boldsymbol{\zeta}$-plane of the $j$-th vortex. The circulation around the body is evaluated via the Kelvin theorem as:

$$
\Gamma_{b}=\Gamma_{b 0}-\sum_{j=1}^{n} \Gamma_{j}
$$

where $\Gamma_{b 0}$ denotes the circulation at the initial time.

\subsection{Kutta condition and vortex method}

The vorticity shed from the trailing edge is modeled as a sequence of vortices, whose circulations are determined by imposing the Kutta condition at the shedding time. Although using a vortex method makes the 
aerodynamic model semi-analytical, because the free-wake dynamics has to be numerically simulated by means of a time-marching procedure, nevertheless this enables to remove the flat-wake assumption common to all the linearized analytical models.

The generation of the nascent vortex is implemented as follows. At each time-step, a new vortex is placed at a point close to the trailing-edge $\left(\boldsymbol{x}_{+}\right.$, which is mapped onto $\left.\boldsymbol{\zeta}_{+}=+R\right)$. The nascent-vortex position in the $\zeta$-plane is assumed as $\zeta^{\star}=R(1+\delta)$, where the offset $\delta>0$ is small with respect to 1 and fixed depending on the maximum distance traveled by the vortices in a time step. The nascent-vortex circulation $\Gamma^{\star}$ is determined by imposing the transformed conjugate velocity $\partial_{\boldsymbol{\zeta}} \tilde{\boldsymbol{w}}$ to vanish at the point $\boldsymbol{\zeta}=+R$, so that the conjugate velocity $\overline{\boldsymbol{u}}=\partial_{\boldsymbol{\zeta}} \tilde{\boldsymbol{w}} \partial_{\boldsymbol{x}} \boldsymbol{\zeta}$ keeps finite on the trailing edge. Therefore, the Kutta condition is written as:

$$
\left.\partial_{\boldsymbol{\zeta}}\left(\tilde{\boldsymbol{w}}_{\infty}+\tilde{\boldsymbol{w}}_{d}+\tilde{\boldsymbol{w}}_{c}^{\star}+\tilde{\boldsymbol{w}}_{w}^{\star}\right)\right|_{\zeta=+R}+\frac{\Gamma^{\star}}{2 \pi \boldsymbol{i}}\left\{\frac{1}{R-R(1+\delta)}-\frac{1}{R-R^{2} /[R(1+\delta)]}+\frac{1}{R}\right\}=0,
$$

where the circulation and wake complex potentials denoted by $\tilde{\boldsymbol{w}}_{c}^{\star}$ and $\tilde{\boldsymbol{w}}_{w}^{\star}$ do not include the contribution due to the nascent vortex. Note that the conjugate velocity $\overline{\boldsymbol{u}}(\boldsymbol{\zeta} ; t)$ still has a first order infinite on the leading edge $\left(\boldsymbol{x}_{-}\right.$, mapped onto $\left.\boldsymbol{\zeta}_{-}=-R\right)$. As a consequence, the aerodynamic force and moment have to be evaluated also using Cauchy principal value integrals.

The vortex velocities in the physical and transformed planes are given by:

$$
\dot{\boldsymbol{x}}_{j}=\left.\overline{\partial_{\boldsymbol{\zeta}} \tilde{\boldsymbol{w}} \partial_{\boldsymbol{x}} \boldsymbol{\zeta}}\right|_{\boldsymbol{\zeta}=\boldsymbol{\zeta}_{j}}, \quad \dot{\boldsymbol{\zeta}}_{j}=\left.\left(\dot{\boldsymbol{x}}_{j}-\partial_{t} \boldsymbol{x}\right) \partial_{\boldsymbol{x}} \boldsymbol{\zeta}\right|_{\boldsymbol{\zeta}=\boldsymbol{\zeta}_{j}},
$$

where the transformed conjugate velocity at each vortex location is evaluated as:

$$
\left.\partial_{\zeta} \tilde{\boldsymbol{w}}\right|_{\boldsymbol{\zeta}=\boldsymbol{\zeta}_{j}}=\left.\partial_{\boldsymbol{\zeta}}\left(\tilde{\boldsymbol{w}}_{\infty}+\tilde{\boldsymbol{w}}_{d}+\tilde{\boldsymbol{w}}_{c}\right)\right|_{\boldsymbol{\zeta}=\boldsymbol{\zeta}_{j}}+\frac{1}{2 \pi \boldsymbol{i}} \sum_{k=1}^{n} \Gamma_{k}\left(\frac{\overline{\boldsymbol{\zeta}}_{j}-\overline{\boldsymbol{\zeta}}_{k}}{\left|\boldsymbol{\zeta}_{j}-\boldsymbol{\zeta}_{k}\right|^{2}+\varepsilon^{2}}-\frac{1}{\boldsymbol{\zeta}_{j}-R^{2} / \overline{\boldsymbol{\zeta}}_{k}}+\frac{1}{\boldsymbol{\zeta}_{j}}\right)
$$

The first term in the vortex-induced contribution in Eq. (18) is given by the desingularized Biot-Savart kernel in complex form (Refs. [29, 30]):

$$
\boldsymbol{K}_{\varepsilon}(\boldsymbol{x}):=-\frac{1}{2 \pi \boldsymbol{i}} \frac{\boldsymbol{x}}{|\boldsymbol{x}|^{2}+\varepsilon^{2}},
$$

which corresponds to a desingularized Green function and a modified vorticity field (see App. A). This smoothing procedure has the effect of regularizing local instabilities in the wake arising from the point-vortex approximation, thus allowing for a better integration of the free-wake dynamics without affecting the aerodynamic loads for appropriate values of the parameter $\varepsilon$. However, the formulation proposed for unsteady aerodynamics is rather general and could use a different vortex method and/or regularization of the Biot-Savart kernel.

\subsection{Aerodynamic loads}

The aerodynamic loads are obtained by specializing Eqs. (5) and (8) to the case of a flat-plate airfoil using the relations deduced in the preceding subsections. The integrals are evaluated via the residue theorem and, because of the leading-edge singularity, also using Cauchy principal value integrals. 
Introduced the following (time-dependent) coefficients depending on the wake geometry:

$$
\begin{aligned}
& \boldsymbol{a}^{(k)}= a_{x}^{(k)}+\boldsymbol{i} a_{y}^{(k)}:=R^{k} \sum_{j=1}^{n} \frac{\Gamma_{j}}{\boldsymbol{\zeta}_{j}^{k}} \\
& \boldsymbol{b}^{\mp}= b_{x}^{\mp}+\boldsymbol{i} b_{y}^{\mp}:=R \sum_{j=1}^{n} \frac{\Gamma_{j}}{\boldsymbol{\zeta}_{j} \pm R} \\
& \boldsymbol{c}^{\mp}=c_{x}^{\mp}+\boldsymbol{i} c_{y}^{\mp}:=2 R^{2} \sum_{j, k=1}^{n} \frac{\Gamma_{j} \Gamma_{k}}{\left(\overline{\boldsymbol{\zeta}}_{j} \pm R\right)\left(\boldsymbol{\zeta}_{k}-R^{2} / \overline{\boldsymbol{\zeta}}_{j}\right)} \\
& \boldsymbol{d}=d_{x}+\boldsymbol{i} d_{y}:=2 R^{2} \sum_{j, k=1}^{n} \frac{\Gamma_{j} \Gamma_{k}}{\overline{\boldsymbol{\zeta}}_{j}\left(\boldsymbol{\zeta}_{k}-R^{2} / \overline{\boldsymbol{\zeta}}_{j}\right)},
\end{aligned}
$$

the normal component of the aerodynamic force (divided by $\rho$ ) is written as:

$$
\begin{aligned}
\frac{F_{n}^{(a)}}{\rho}= & -\frac{\pi}{4} l^{2} \dot{V}_{n}+\left[-u_{\infty} \cos (\alpha+\beta)-V_{\tau}+\frac{b_{y}^{-}+b_{y}^{+}}{\pi l}\right] \Gamma_{b}+ \\
& +\frac{\pi}{4} l^{2}\left[\dot{u}_{\infty} \sin (\alpha+\beta)+(\dot{\alpha}+\dot{\beta}) u_{\infty} \cos (\alpha+\beta)\right]-\frac{l}{2} \dot{a}_{x}^{(1)}+ \\
& +\left(b_{y}^{-}-b_{y}^{+}\right)\left[V_{n}-u_{\infty} \sin (\alpha+\beta)\right]+\frac{l \dot{\alpha}}{4}\left(b_{y}^{-}+b_{y}^{+}-2 a_{y}^{(1)}\right)+ \\
& +\frac{1}{2 \pi l}\left(2 b_{x}^{-} b_{y}^{-}-2 b_{x}^{+} b_{y}^{+}+c_{y}^{+}-c_{y}^{-}\right) \\
=: & \frac{\pi}{4} l^{2}\left(G_{n}-\dot{V}_{n}\right) .
\end{aligned}
$$

The quantity $\dot{V}_{n}$ in Eq. (21) depends on the normal plate-center acceleration and leads to the identification of the added mass $\pi l^{2} \rho / 4$, namely the mass (per unit span length) of the fluid accelerated by the plate. The tangent component of the force (divided by $\rho$ ) is proportional to a quadratic polynomial in $\Gamma_{b}$ :

$$
\frac{F_{\tau}^{(a)}}{\rho}=\frac{\Gamma_{b}^{2}-2 q_{1} \Gamma_{b}+q_{2}}{4 \pi l}=: \frac{\pi}{4} l^{2} G_{\tau},
$$

where the coefficients $q_{1}$ and $q_{2}$ are:

$$
\begin{aligned}
q_{1}= & -\pi l u_{\infty} \sin (\alpha+\beta)+\pi l V_{n}-\frac{\pi}{4} l^{2} \dot{\alpha}+2 b_{x}^{+} \\
q_{2}= & \pi^{2} l^{2} u_{\infty}^{2} \sin ^{2}(\alpha+\beta)+\frac{\pi^{2}}{16} l^{2}\left(16 V_{n}^{2}-24 l \dot{\alpha} V_{n}+l^{2} \dot{\alpha}^{2}\right)+\frac{\pi^{2}}{2} l^{3} \dot{\alpha} u_{\infty} \sin (\alpha+\beta)+ \\
& +\pi^{2} l^{3} V_{n} \dot{\alpha}-2 \pi^{2} l^{2} V_{n} u_{\infty} \sin (\alpha+\beta)-4 \pi l b_{x}^{+} u_{\infty} \sin (\alpha+\beta)+ \\
& +4 \pi l V_{n} b_{x}^{+}-\pi l^{2} \dot{\alpha} b_{x}^{+}+2\left(b_{x}^{-2}+b_{y}^{-2}+b_{x}^{+2}-b_{y}^{+2}+c_{x}^{+}-c_{x}^{-}\right) .
\end{aligned}
$$

Note that, according to Eq. (1), the aerodynamic force should be normal to a flat-plate airfoil, because an inviscid fluid applies a purely normal pressure stress on the plate boundary. However, the tangent component of the force given by Eq. (22) is not exactly zero, even though it is very small compared the normal one. A new shedding criterion could be therefore obtained by imposing the tangent aerodynamic force to vanish. Indeed, it can been verified (analytically in steady cases and numerically in presence of wake) that the resulting quadratic equation in $\Gamma_{b}$ would give two real and coincident roots. This equation could be used to evaluate the circulation around the body at each time step, then computing the nascent-vortex circulation via the Kelvin theorem. 
The aerodynamic moment with respect to the origin is recast as $M^{(a)}(0)=-H_{\tau} F_{n}^{(a)}+H_{n} F_{\tau}^{(a)}+M^{(a)}(\boldsymbol{H})$, where the one about the plate center (divided by $\rho$ ) is given by :

$$
\begin{aligned}
\frac{M^{(a)}(\boldsymbol{H})}{\rho}= & \frac{\pi}{128} l^{4} \ddot{\alpha}+\frac{\pi}{4} l^{2}\left[V_{n}-u_{\infty} \sin (\alpha+\beta)\right]\left[V_{\tau}+u_{\infty} \cos (\alpha+\beta)\right]+ \\
+ & \frac{l}{2}\left(a_{y}^{(1)}-b_{y}^{-}-b_{y}^{+}\right)\left[V_{n}-u_{\infty} \sin (\alpha+\beta)\right]+\frac{l}{2}\left[V_{\tau}+u_{\infty} \cos (\alpha+\beta)\right] a_{x}^{(1)}+ \\
& -\frac{l^{2}}{16}\left(\dot{a}_{x}^{(2)}+2 \dot{\alpha} a_{y}^{(2)}\right)+\left(\frac{\Gamma_{b}}{2 \pi}+\frac{\dot{\alpha} l^{2}}{8}\right)\left(b_{y}^{+}-b_{y}^{-}\right)+ \\
& +\frac{1}{4 \pi}\left(-2 b_{x}^{-} b_{y}^{-}-2 b_{x}^{+} b_{y}^{+}+c_{y}^{-}+c_{y}^{+}-d_{y}\right) \\
= & \frac{\pi}{128} l^{4}\left(\ddot{\alpha}-\mathcal{M}^{(a)}\right) .
\end{aligned}
$$

The pitch acceleration $\ddot{\alpha}$ appears in Eq. (23), so identifying the added moment of inertia $\pi l^{4} \rho / 128$.

\section{Aeroelastic model}

An aeroelastic model for the flat-plate airfoil depicted in Fig. 1 is derived. The section moves under the aerodynamic loads and elastic reactions. The mass and elastic centers are here assumed at the plate center, but the model can be easily generalized to include the offsets. The section is restrained from bending along the $x$ and $y$-axes and from torsion by linear springs of constants $k_{x}=m\left(2 \pi f_{x}\right)^{2}, k_{y}=m\left(2 \pi f_{y}\right)^{2}$ and $k_{\alpha}=J_{\alpha}\left(2 \pi f_{\alpha}\right)^{2}$, where $m$ and $J_{\alpha}$ denote the mass and the moment of inertia with respect to the elastic center per unit span length, respectively. The $x$ - and $y$-components of the elastic force and the elastic moment are given by $F_{x}^{(e)}=$ $-k_{x}\left(H_{x}-H_{x e}\right), F_{y}^{(e)}=-k_{z}\left(H_{y}-H_{y e}\right)$ and $M^{(e)}=k_{\alpha}\left(\alpha-\alpha_{e}\right)$, where $\boldsymbol{H}_{e}=H_{x e}+\boldsymbol{i} H_{y e}$ and $\alpha_{e}$ are the plate-center position and the angle of elastic equilibrium (namely of vanishing elastic reactions). Once the ratios between the added mass and moment of inertia and the section ones $\sigma=\pi l^{2} \rho /(4 m)$ and $\mu=\pi l^{4} \rho /\left(128 J_{\alpha}\right)$ are introduced, the equations of motion are given by:

$$
\ddot{\boldsymbol{H}}=\sigma\left[\left(G_{n}-\dot{V}_{n}\right) \boldsymbol{n}+G_{\tau} \boldsymbol{\tau}\right]+\frac{\boldsymbol{F}^{(e)}}{m}, \quad \ddot{\alpha}=\mu\left(\mathcal{M}^{(a)}-\ddot{\alpha}\right)-\frac{M^{(e)}}{J_{\alpha}} .
$$

The quantity $\dot{V}_{n}$ is evaluated from the first of the equations above (using $\dot{\boldsymbol{n}}=-\dot{\alpha} \boldsymbol{\tau}$ ):

$$
\dot{V}_{n}=\ddot{\boldsymbol{H}} \cdot \boldsymbol{n}-\dot{\alpha} \dot{\boldsymbol{H}} \cdot \boldsymbol{\tau}=\frac{1}{1+\sigma}\left(\sigma G_{n}+\frac{F_{n}^{(e)}}{m}-\dot{\alpha} V_{\tau}\right)
$$

and substituted into the same equation. Once the initial data $\boldsymbol{H}_{0}, \dot{\boldsymbol{H}}_{0}, \alpha_{0}$ and $\dot{\alpha}_{0}$ are given, the Cauchy problem for the plate motion follows:

$$
\left\{\begin{aligned}
\ddot{\boldsymbol{H}} & =\frac{1}{1+\sigma}\left[\sigma\left(G_{n}+\dot{\alpha} V_{\tau}\right)+\frac{F_{n}^{(e)}}{m}\right] \boldsymbol{n}+\left(\sigma G_{\tau}+\frac{F_{\tau}^{(e)}}{m}\right) \boldsymbol{\tau} \\
\ddot{\alpha} & =\frac{1}{1+\mu}\left(\mu \mathcal{M}^{(a)}-\frac{M^{(e)}}{J_{\alpha}}\right) \\
\boldsymbol{H}(0) & =\boldsymbol{H}_{0}, \dot{\boldsymbol{H}}(0)=\dot{\boldsymbol{H}}_{0}, \alpha(0)=\alpha_{0}, \dot{\alpha}(0)=\dot{\alpha}_{0} .
\end{aligned}\right.
$$




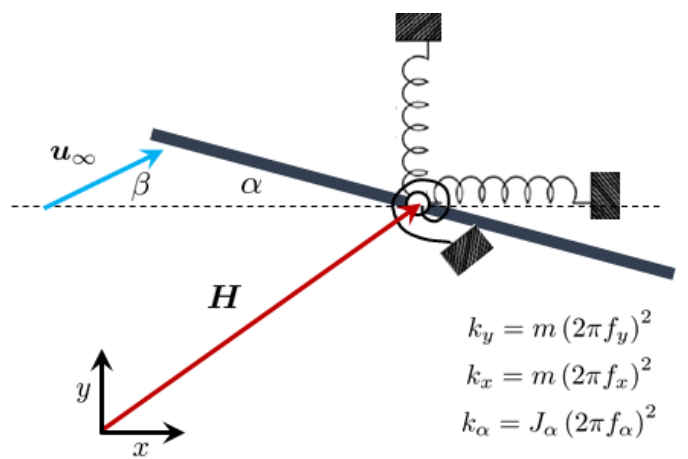

Figure 1: Aeroelastic model of a flat-plate airfoil.

Under the action of the aerodynamic and elastic loads, the plate reaches the equilibrium configuration (equilibrium quantities are denoted by a tilde):

$$
\left\{\begin{aligned}
\tilde{H}_{x} & =H_{x e}+\frac{\sigma u_{\infty}^{2}}{2 \pi^{2} f_{x}^{2} l} \sin \tilde{\alpha} \sin [2(\tilde{\alpha}+\beta)] \\
\tilde{H}_{y} & =H_{y e}+\frac{\sigma u_{\infty}^{2}}{2 \pi^{2} f_{y}^{2} l} \cos \tilde{\alpha} \sin [2(\tilde{\alpha}+\beta)] \\
\tilde{\alpha} & =\alpha_{e}+\frac{4 \mu u_{\infty}^{2}}{\pi^{2} f_{\alpha}^{2} l^{2}} \sin [2(\tilde{\alpha}+\beta)]
\end{aligned}\right.
$$

having used the stationary body circulation $\tilde{\Gamma}_{b}=-\pi l u_{\infty} \sin (\tilde{\alpha}+\beta)$. Once the steady-state angle of attack $\tilde{\alpha}$ is deduced from the third equation, the first two equations give the stationary plate-center position.

\section{Results}

A numerical integration of the model is performed using a fourth-order Runge-Kutta scheme. An adaptive time increment is adopted in order to enable a robust integration of the body motion and free-wake dynamics. Since a new vortex is generated at each integration step, the wake shedding rate is adaptive as well. The time increment is varied during the time-marching procedure according to an imposed threshold for the body and vortices displacements. At each step, the maximum vortex velocity (in modulus) is compared with the translational and rotational body velocities to identify the overall maximum velocity. The current time increment is then computed so that its product by the maximum velocity equals the imposed threshold for the displacements. Appropriate values for the latter are found for each test case via a convergence study. The sensitivity of the solution with respect to $\delta$ and $\varepsilon$ is also examined. The parameter $\delta$, which identifies the nascent-vortex initial position in the $\boldsymbol{\zeta}$-plane, is fixed such that the corresponding point in the $\boldsymbol{x}$-plane has an offset from the trailing edge lower than the threshold for the displacements. Provided this, the solution is basically insensitive to $\delta$. Concerning the smoothing parameter $\varepsilon$, values of the order of $10^{-1}$ are found to improve the wake integration in the present simulations without affecting the aerodynamic loads.

The numerical results are discussed below. Validation of the unsteady aerodynamic model is first carried out. Then, the aeroelastic model is used to study the response of a flat-plate airfoil to a sudden start of the flow and to the interaction with a passing vortex disturbing a steady-state configuration. 


\subsection{Validation with linearized models}

In this subsection, the unsteady aerodynamic model is validated with the linearized solutions by Wagner (sudden start at fixed angle of attack, Ref. [1]), Theodorsen (small-amplitude plunge and pitch in steady horizontal flow, Refs. [3, 4]), and Isaacs (sinusoidally-varying free-stream velocity at fixed angle of attack, Ref. [7]).

\subsubsection{Wagner Problem}

A first validation of the aerodynamic model is achieved by simulating the transient lift response to a sudden start from rest in steady horizontal flow at fixed angle of attack. With the aim to recover the Wagner solution (Ref. [1]), the simulation is performed in the linear range at $\alpha_{0}=1^{\circ}$. The resulting lift time-history, normalized by the steady-state value $L_{0}$, is compared with the Wagner function in Fig. 2 . Time is normalized by $l /\left(2 u_{\infty}\right)$. The obtained lift response is found to match the theoretical curve, in particular capturing the instantaneous jump of the non-dimensional lift to 0.5 at $t=0^{+}$.

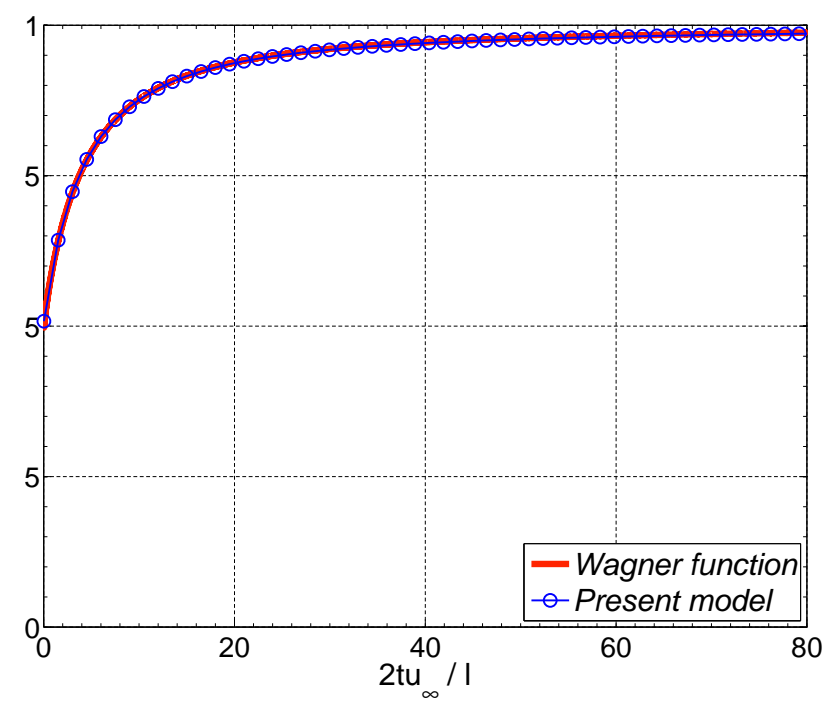

Figure 2: Wagner problem: non-dimensional lift $L / L_{0}$ due to a sudden start from rest at fixed angle of attack $\alpha_{0}=1^{\circ}$.

\subsubsection{Validation with the Theodorsen model}

The unsteady aerodynamic model is here validated with the linearized solution by Theodorsen (Refs. [4, 3]) for the case of small-amplitude plunge and pitch in steady horizontal flow. The validation is first carried out analytically in order to show that the present formulation reduces to the Theodorsen one in a linearized framework. A numerical example is then considered to test the implementation of the aeroelastic model.

Since the wake is modeled as a sequence of vortices rather than a continuous vortex sheet in the present formulation, the reference linearized solution is the one in Ref. [4] (Ch. 5, Eqs. (5.271), (5.272), (5.289), and (5.290) 
with $a=0$ to have the plate-center displacement as degree of freedom as in the present model):

$$
\begin{aligned}
L & =\frac{\pi}{4} \rho l^{2}\left(-\ddot{H}_{y}+u_{\infty} \dot{\alpha}\right)+\frac{\rho u_{\infty} \Gamma_{0} \xi_{0}}{\sqrt{\xi_{0}^{2}-l^{2} / 4}}, \\
M_{y} & =\frac{\pi}{4} \rho l^{2}\left(u_{\infty} \dot{H}_{y}-u_{\infty}^{2} \alpha+\ddot{\alpha} l^{2} / 32\right)+\frac{l^{2}}{8} \frac{\rho u_{\infty} \Gamma_{0}}{\sqrt{\xi_{0}^{2}-l^{2} / 4}} .
\end{aligned}
$$

Equation (26) holds for arbitrary motions and gives the linearized lift $L$ and pitching moment $M_{y}$ (about the plate center) due to non-circulatory effects and to a single point vortex of circulation $\Gamma_{0}$ and position along the $x$-axis $\xi_{0}$, flowing downstream with velocity $u_{\infty}$. Note that the aerodynamic loads in Eq. (26) are written assuming upward-positive vertical displacements and counterclockwise-positive pitching moments, in contrast with Ref. [4].

Following Theodorsen, the assumptions of small angle of attack, steady horizontal flow, and geometricallyprescribed flat wake are made to simplify Eqs. (21) and (23) in order to recover Eq. (26). The linearized lift and pitching moment given by the present model turn out to be:

$$
\begin{aligned}
L & =\frac{\pi}{4} \rho l^{2}\left(-\ddot{H}_{y}+u_{\infty} \dot{\alpha}\right)-\rho u_{\infty} \Gamma_{b}-\rho \frac{l}{2} \dot{a}_{x}^{(1)} \\
M_{y} & =\frac{\pi}{4} \rho l^{2}\left(u_{\infty} \dot{H}_{y}-u_{\infty}^{2} \alpha+\ddot{\alpha} l^{2} / 32\right)+\rho u_{\infty} \frac{l}{2} a_{x}^{(1)}-\rho \frac{l^{2}}{16} \dot{a}_{x}^{(2)} .
\end{aligned}
$$

The above results are obtained by considering that $V_{n} \approx \dot{H}_{y}, \dot{V}_{n} \approx \ddot{H}_{y}$, and $V_{\tau} \approx 0$ in the small-angle approximation, and that the wake coefficients in Eq. (20) are purely real when flat wake is assumed.

To recover the Theodorsen solution in Eq. (26), the quantities $a_{x}^{(1)}, \dot{a}_{x}^{(1)}$, and $\dot{a}_{x}^{(2)}$ are rewritten in terms of vortex positions and velocities in the physical plane rather than in the transformed one. This is achieved by observing that the present conformal map given by Eq. (9) reduces to the usual stationary Joukowski map in the case of small disturbances, so that one has $(R=l / 4)$ :

$$
\zeta_{x j}=\frac{1}{2}\left(\xi_{j}+\sqrt{\xi_{j}^{2}-l^{2} / 4}\right), \quad \dot{\zeta}_{x j}=u_{\infty} \frac{\zeta_{x j}^{2}}{\zeta_{x j}^{2}-l^{2} / 16} .
$$

Substituting the above relations and using Kelvin theorem in Eq. (27), with some manipulation it reduces to Eq. (26) (the details are reported in App. B).

Next, the numerical implementation of the aeroelastic model is tested by verifying the stability margin predicted by Theodorsen for a given set of system parameters. This is accomplished by simulating the aeroelastic free response of a flat-plate airfoil due to a small initial perturbation (like an imposed pitch rate) at zero angle of attack below and above the expected flutter speed. The non-dimensional parameters assumed for the computations are $\sigma=0.1, \mu=0.05,\left(f_{h} / f_{\alpha}\right)^{2}=0.5$, with the mass and elastic centers at the plate center. The corresponding non-dimensional flutter speed is estimated by Theodorsen as $u_{F} /\left(l \pi f_{\alpha}\right)=1.41$ (Ref. [4], Ch. 5, p. 543, Fig. (9.5D)). Two simulations are then performed for $u_{\infty}=0.97 u_{F}$ and $u_{\infty}=1.02 u_{F}$. In both cases, a non-dimensional initial pitch rate $\dot{\alpha} l /\left(2 u_{\infty}\right)=0.001$ is applied to disturb the section from the trivial aeroelastostatic solution (zero angle of attack). The resulting time-histories of the angle of attack and of the non-dimensional plate-center plunge $\left(H_{y}\right.$ is normalized by $\left.l / 2\right)$ are plotted versus the non-dimensional time in Fig. 3. In agreement with the theoretical prediction, the system is found to be stable at the lower velocity but unstable at the higher one. 


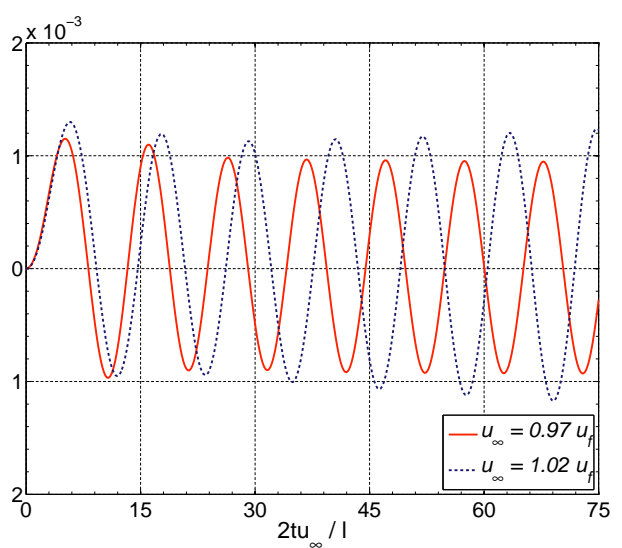

(a)

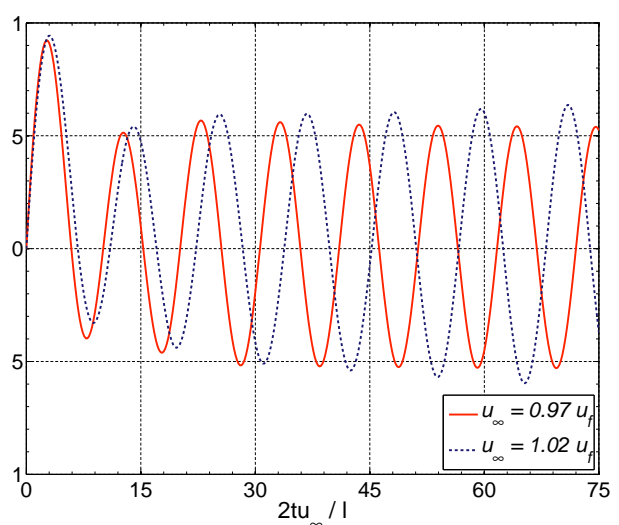

(b)

Figure 3: Stability margin: time-histories of the non-dimensional plunge at the plate center $(a)$ and of the angle of attack $(b)$ for $u_{\infty}=0.97 u_{F}$ and $u_{\infty}=1.02 u_{F}$.

\subsubsection{Sinusoidally-varying free-stream velocity}

To complete the model assessment against classical linearized solutions, the case of a sinusoidally-varying horizontal free stream is finally explored. The case study proposed is the one in Ref. [25], comparing the results by the free-wake formulation of Yan et al. with the linearized predictions by Isaacs (Ref. [7]), Peters (Ref. [14]), and Greenberg (Ref. [8]). The plate experiences the steady-state lift due to a fixed angle of attack $\alpha_{0}=5^{\circ}$ (in the linear range) plus the transient lift variation due to a time-dependent asymptotic velocity $u_{\infty}(t)=u_{\infty 0}[1+\lambda \sin (\omega t)]$, with $\lambda=0.8$ and $k:=\omega l /\left(2 u_{\infty 0}\right)=0.2$. The lift time-history by the present model, normalized by the steady-state value $L_{0}$, is plotted versus the phase $\omega t$ and compared with the other predictions in Fig. 4. The present numerical results are found to match very well with the other ones.

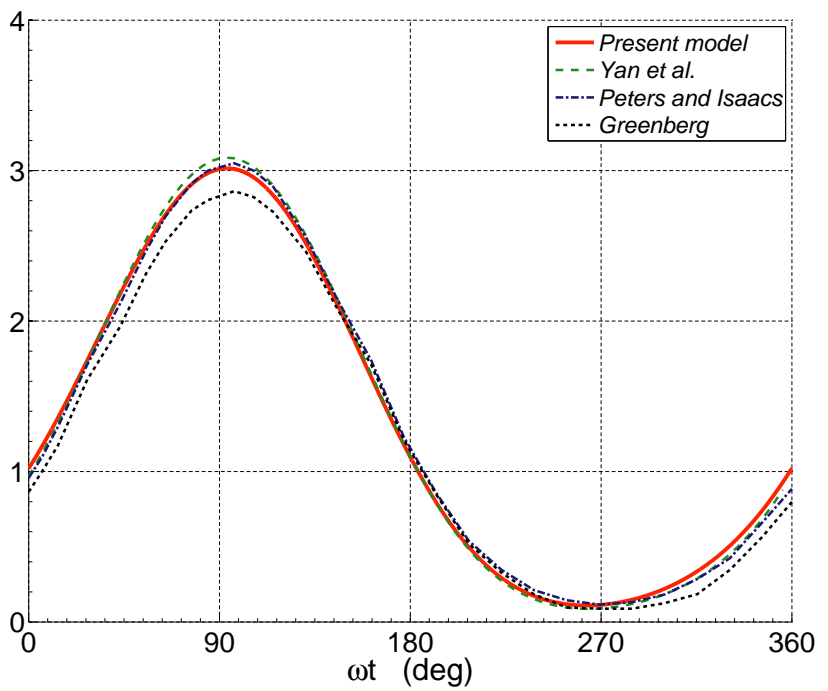

Figure 4: Sinusoidally-varying free-stream velocity: non-dimensional lift $L / L_{0}$ with $\alpha_{0}=5^{\circ}$ compared with the solutions by Isaacs (Ref. [7]), Peters (Ref. [14]), Greenberg (Ref. [8]), and Yan et al. (Ref. [25]). 


\subsection{Validation with experimental results}

The unsteady aerodynamic model is here assessed with experimental data and theoretical predictions for an imposed large-amplitude maneuver. Specifically, the canonical pitch-up, hold, pitch-down motion introduced by the AIAA Fluid Dynamics Technical Committee's Low Reynolds Number Discussion Group (Ref. [32]) and illustrated in Fig. 5 is considered.

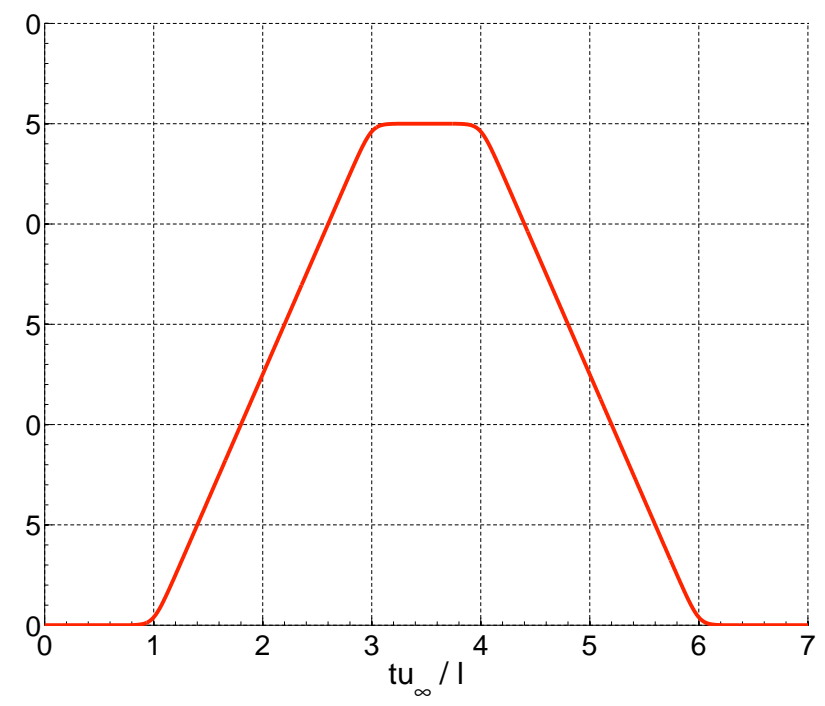

Figure 5: Canonical pitch-up, hold, pitch-down maneuver up to $\alpha=25^{\circ}$ (Ref. [32]).

The lift coefficient predicted by the present model is compared with the water-tunnel experimental data and theoretical prediction by Ramesh et al. (Ref. [16]) in Figs. 6, 7, and 8 for the three cases of pivot about the leading edge, half-chord, and trailing edge. For the case of half-chord pivot, the present numerical results are also compared with the simulation by Yan et al. (Ref. [25]). The time-histories are plotted versus the chordbased non-dimensional time $t u_{\infty} / l$, with $l=0.0762 \mathrm{~m}$ and $u_{\infty}=0.066 \mathrm{~m} / \mathrm{s}$ as in the water-tunnel experiments (Ref. [16]). The present model matches well the experimental data during the upstroke. Discrepancies are found between the predicted and experimental lift coefficients during the hold and downstroke, which can be explained by considering that leading-edge vortex shedding is neglected in the present formulation. However, the qualitative behavior of the lift coefficient is still captured. Moreover, note that the present numerical results are in agreement with the model by Ramesh et al. (Ref. [16]) and match the free-wake simulation performed by Yan et al. (Ref. [25]) for the case of half-chord pivot.

\subsection{Aeroelastic response to a sudden start}

Once the fidelity of the present model has been demonstrated by means of the preceding validations, the aeroelastic response of a flat-plate airfoil to a sudden start of the flow is here discussed.

In order to simulate a sudden start, the section is assumed to be in elastic equilibrium at the initial time $\left(\boldsymbol{H}_{0}=\boldsymbol{H}_{e}, \alpha_{0}=\alpha_{e}, \Gamma_{b 0}=0\right)$ and the flow is at rest. As the simulation starts, the modulus of the free-stream velocity is then increased as a hyperbolic tangent function up to a stationary value $u_{\infty}$ (below the flutter speed). The free stream is horizontal $\left(\beta \equiv 0^{\circ}\right)$. A set of test cases, listed in Tab. 3, is established to point out influence 


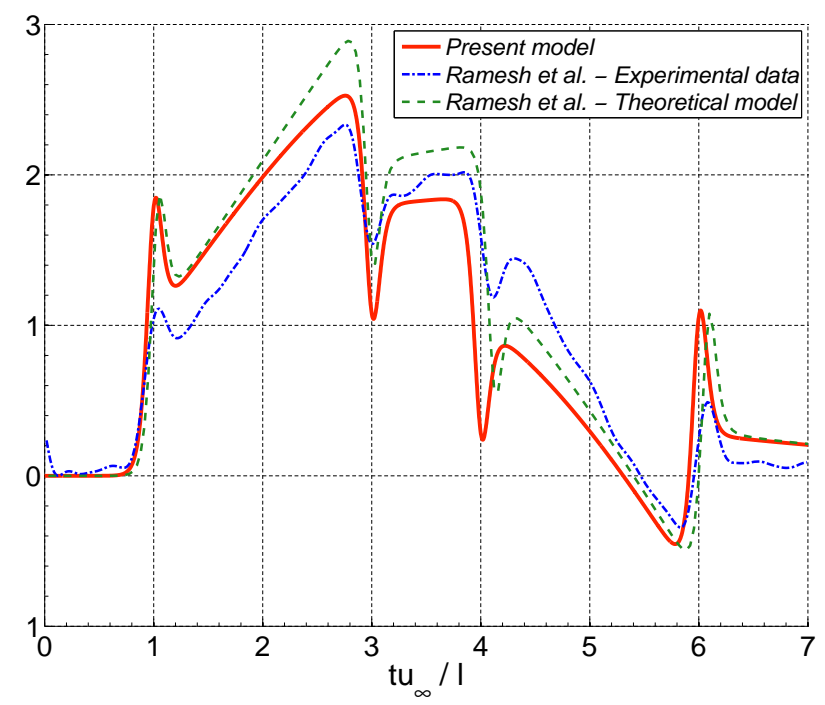

Figure 6: Lift coefficient due to the canonical pitch-up, hold, pitch-down maneuver in Fig. 5 with pivot about the leading edge. Comparison with the experimental data and theoretical prediction by Ramesh et al. (Ref. [16]).

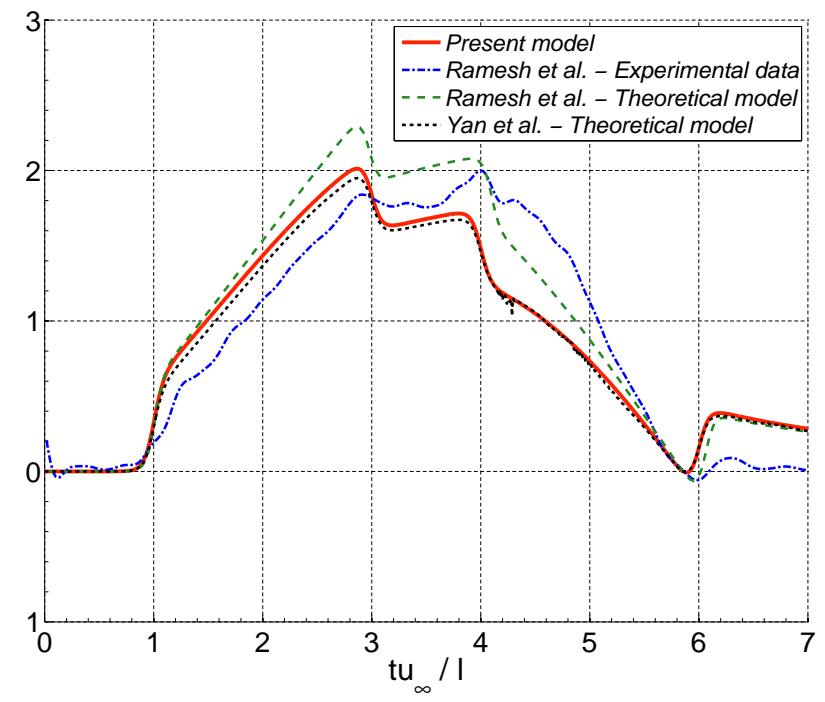

Figure 7: Lift coefficient due to the canonical pitch-up, hold, pitch-down maneuver in Fig. 5 with pivot about the half-chord. Comparison with the experimental data and theoretical prediction by Ramesh et al. (Ref. [16]) and with the theoretical prediction by Yan et al. (Ref. [25]). 


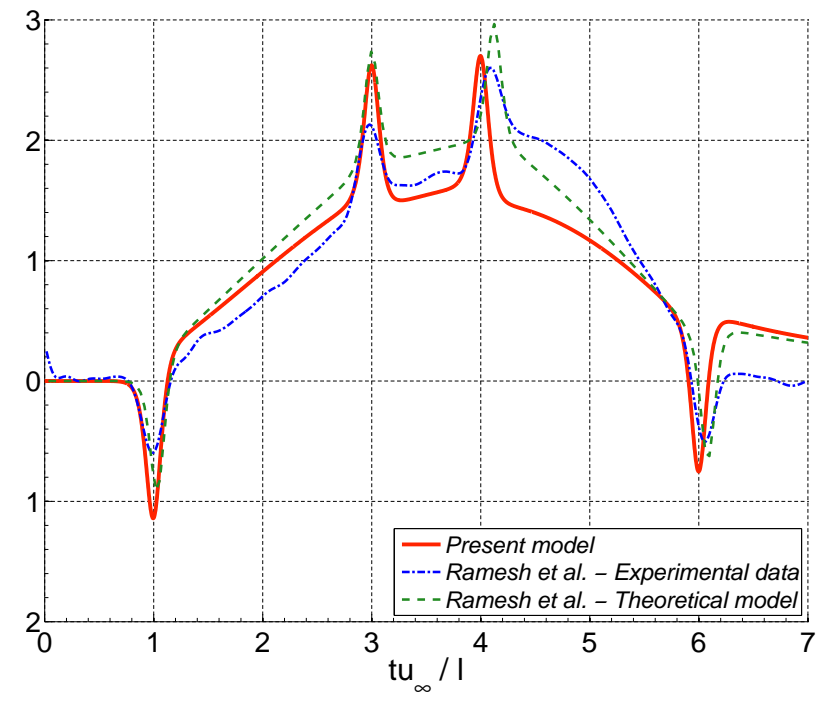

Figure 8: Lift coefficient due to the canonical pitch-up, hold, pitch-down maneuver in Fig. 5 with pivot about the trailing edge. Comparison with the experimental data and theoretical prediction by Ramesh et al. (Ref. [16]).

$\begin{array}{cccccc}\text { Test } & u_{\infty}(\mathrm{m} / \mathrm{s}) & \alpha_{0}(\mathrm{deg}) & \tilde{H}_{x}(\mathrm{~cm}) & \tilde{H}_{y}(\mathrm{~cm}) & \tilde{\alpha}(\mathrm{deg}) \\ \mathbf{1} & 10 & 5 & 0.007 & 1.7 & 5.96 \\ \mathbf{2} & 10 & 10 & 0.027 & 3.2 & 11.87 \\ \mathbf{3} & 15 & 5 & 0.027 & 4.9 & 7.82 \\ \mathbf{4} & 15 & 10 & 0.098 & 9.0 & 15.33\end{array}$

Table 3: Sudden start: free-stream velocity, initial angle of attack, and final configuration for the test cases.

of $u_{\infty}$ and $\alpha_{0}=\alpha_{e}$ on the aeroelastic response. The assumed section parameters are $\sigma=0.1, \mu=0.05$, $H_{x e}=H_{y e}=0, f_{x}=12.5 \mathrm{~Hz}, f_{y}=2.5 \mathrm{~Hz}, f_{\alpha}=5 \mathrm{~Hz}$, and $l=1 \mathrm{~m}$. As for actual wing sections, the surge frequency $f_{x}$ is higher than the plunge frequency $f_{y}$. The horizontal motion and stationary displacement of the plate are thus very small compared to the vertical one, also because the aerodynamic force has a negligible horizontal component for moderate angles of attack. ${ }^{2}$ For this reason, the horizontal motion is not considered in the following discussion. The numerical simulations are carried out using both the fully-nonlinear code and a linearized version of the code, with the aim to point out influence of assuming small angle of attack and geometrically-prescribed flat wake on the prediction of the aeroelastic response.

Figure 9 presents the time-histories of the non-dimensional plunge at the plate center and of the variation in the angle of attack with respect to $\alpha_{0}=\alpha_{e}$ obtained for $\alpha_{0}=5^{\circ}, 10^{\circ}$ with $u_{\infty}=10 \mathrm{~m} / \mathrm{s}($ Tests 1 and 2$)$. Figure 10 compares the results obtained for $u_{\infty}=10 \mathrm{~m} / \mathrm{s}, 15 \mathrm{~m} / \mathrm{s}$ with $\alpha_{0}=5^{\circ}$ (Tests 1 and 3 ). The solid lines in the figures refer to the nonlinear aeroelastic response, whereas the dash-dotted curves to the linearized one (small angle of attack and flat wake). As well known, increasing $u_{\infty}$ or $\alpha_{0}$ reduce the system stiffness, leading

\footnotetext{
${ }^{2}$ Note that neglecting leading-edge vortex shedding limits the field of application of the present model in terms of angle of attack.
} 


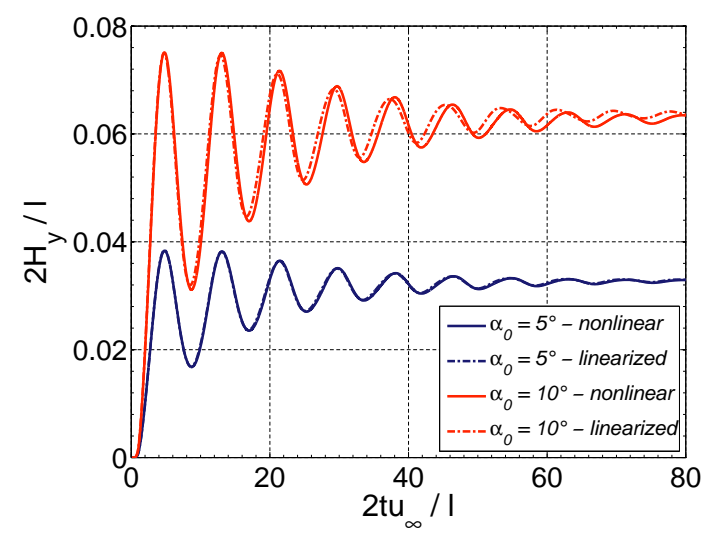

(a)

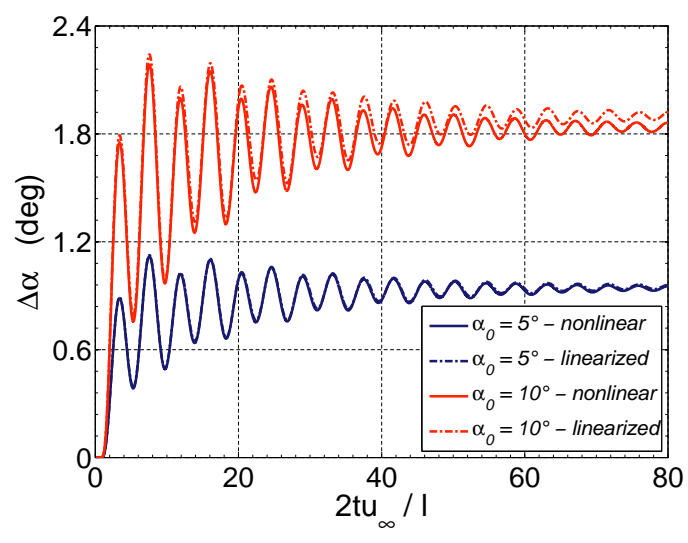

(b)

Figure 9: Sudden start: time-histories of the non-dimensional plunge at the plate center $(a)$ and of the angle of attack variation (b) for $\alpha_{0}=5^{\circ}, 10^{\circ}$ and $u_{\infty}=10 \mathrm{~m} / \mathrm{s}$ (Tests 1 and 2).

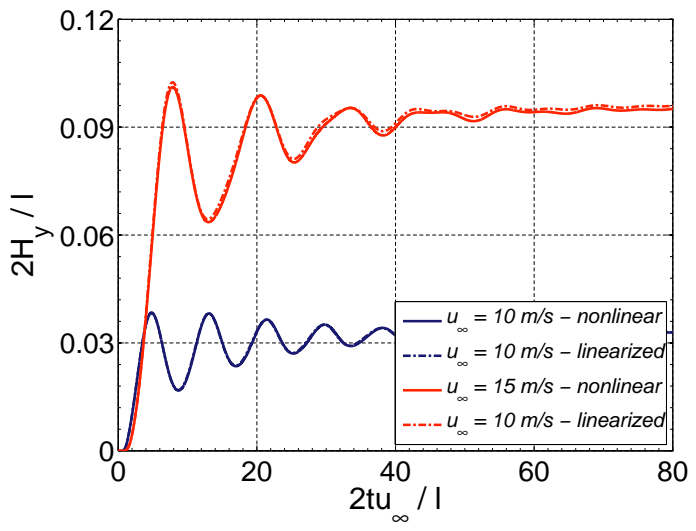

(a)

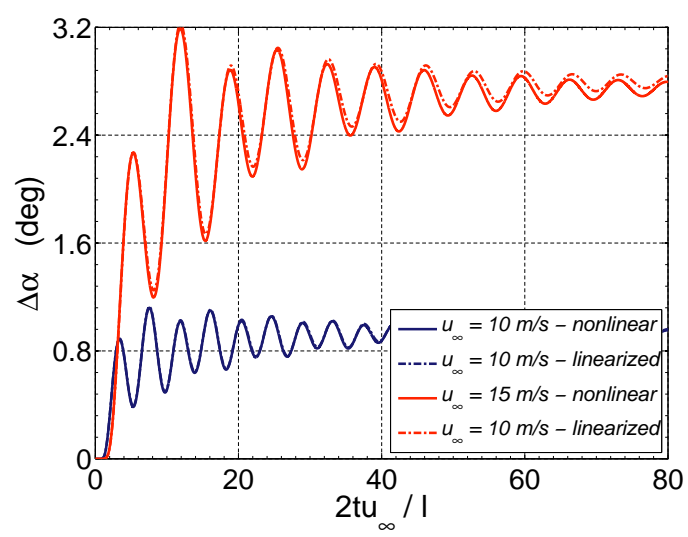

(b)

Figure 10: Sudden start: time-histories of the non-dimensional plunge at the plate center $(a)$ and of the angle of attack variation (b) for $u_{\infty}=10 \mathrm{~m} / \mathrm{s}, 15 \mathrm{~m} / \mathrm{s}$ and $\alpha_{0}=5^{\circ}$ (Tests 1 and 3 ). 


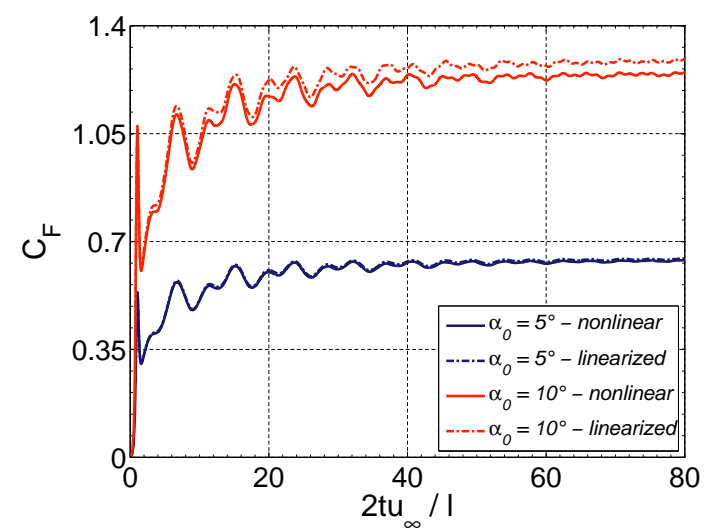

(a)

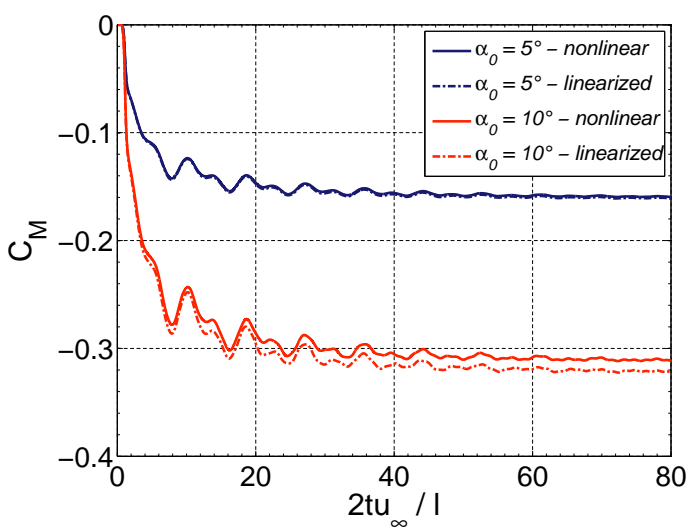

(b)

Figure 11: Sudden start: time-histories of the normal aerodynamic force $(a)$ and pitching moment $(b)$ coefficients for $\alpha_{0}=5^{\circ}, 10^{\circ}$ and $u_{\infty}=10 \mathrm{~m} / \mathrm{s}$ (Tests 1 and 2).

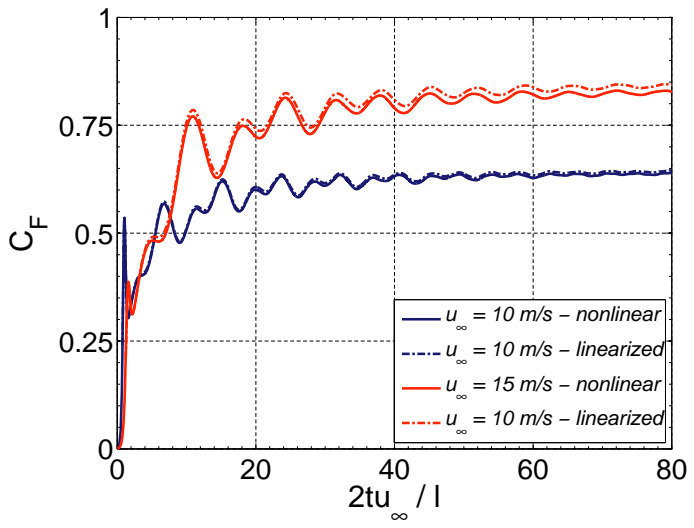

(a)

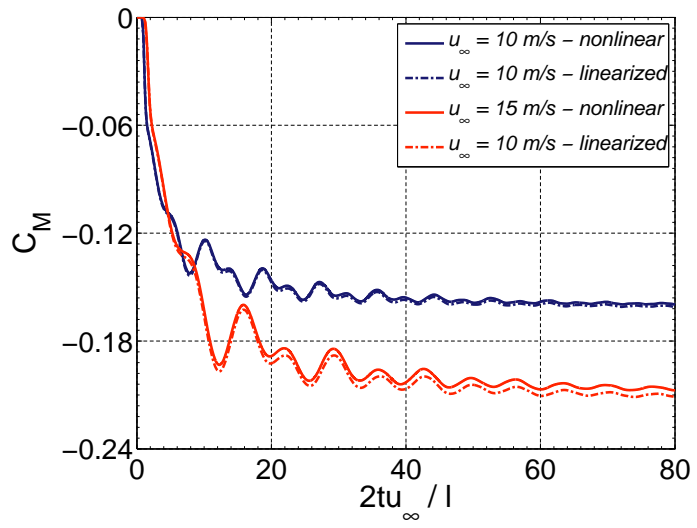

(b)

Figure 12: Sudden start: time-histories of the normal aerodynamic force $(a)$ and pitching moment $(b)$ coefficients for $u_{\infty}=10 \mathrm{~m} / \mathrm{s}$, $15 \mathrm{~m} / \mathrm{s}$ and $\alpha_{0}=5^{\circ}$ (Tests 1 and 3 ).

to larger stationary linear and angular displacements (see Tab. 3), and reduces the aerodynamic damping, causing larger and longer oscillations during the transients. The difference between the linearized and nonlinear predictions is found to increase with the initial angle of attack. Indeed, free-wake effects are more relevant for larger-amplitude pitch motions, due to the fact that the flat-wake assumption is less adequate to describe the actual wake dynamics.

The time-histories of the normal aerodynamic force and pitching moment coefficients are illustrated in Figs. 11 and 12, whereas the non-dimensional tangent is negligible compared to the normal one and it is not presented. The plots show that, as well known, the linearized formulation gives different steady-state aerodynamic loads and thus another aeroelastostatic solution with respect to the nonlinear model due to the assumption of small angle of attack (see Figs. 9 and 10). The time-histories of the non-dimensional location of the center of pressure along the chord are shown in Fig. 13. The center of pressure suddenly leaves the half-chord as the flow starts, then it oscillates close to the quarter-chord during the transient to approach that 


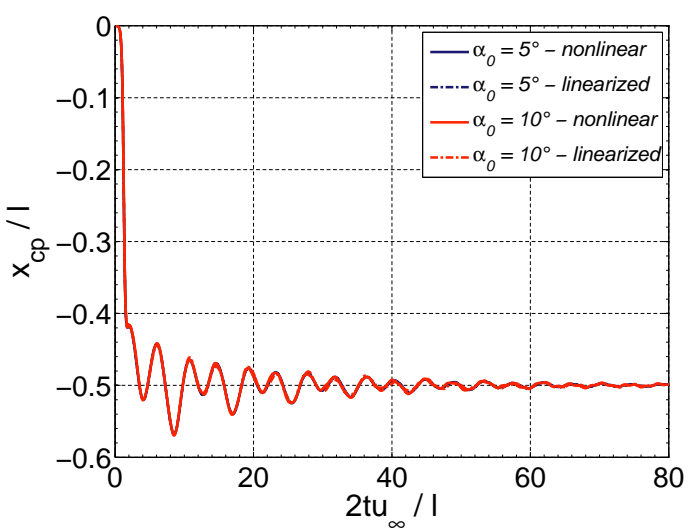

(a)

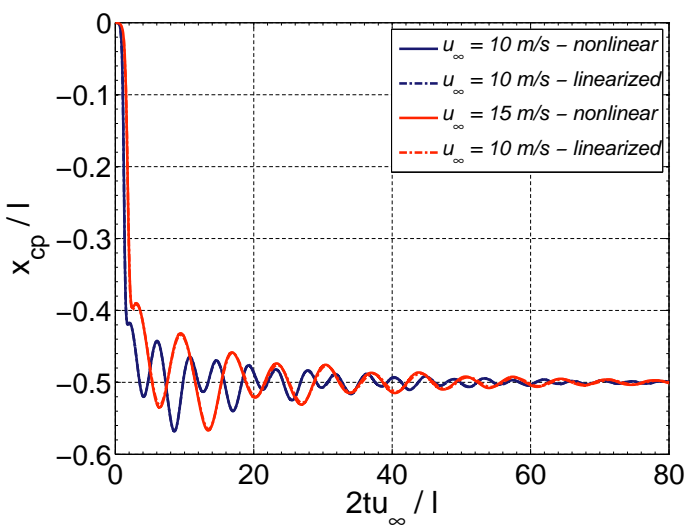

(b)

Figure 13: Sudden start: time-histories of the non-dimensional center of pressure location along the chord for $\alpha_{0}=5^{\circ}, 10^{\circ}$, $u_{\infty}=10 \mathrm{~m} / \mathrm{s}(a)$ and for $u_{\infty}=10 \mathrm{~m} / \mathrm{s}, 15 \mathrm{~m} / \mathrm{s}, \alpha_{0}=5^{\circ}(b)$ (Tests $\left.1-3\right)$.

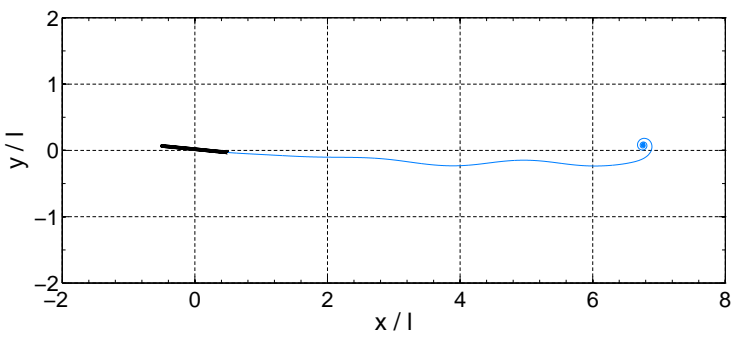

(a)

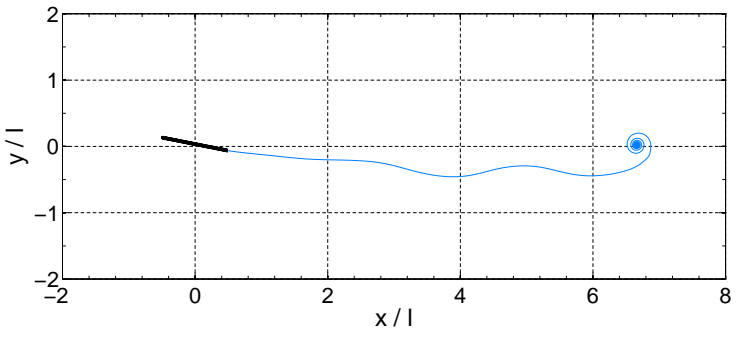

(b)

Figure 14: Sudden start: wake configurations at the non-dimensional time $2 t u_{\infty} / l=14$ for $\alpha_{0}=5^{\circ}(a), 10^{\circ}(b)$ and $u_{\infty}=10 \mathrm{~m} / \mathrm{s}$ (Tests 1 and 2).

point as steady conditions are reached.

Finally, some wake geometries at three different times are shown in Figs. 14 and 15 for $\alpha_{0}=5^{\circ}, 10^{\circ}$ and $u_{\infty}=10 \mathrm{~m} / \mathrm{s}$ (Tests 1 and 2). Spatial coordinates are in unit chord. Figure 14 shows that the steep gradient in the shed vorticity as the flow starts results in a starting vortex, whose strength increases with $\alpha_{0}$. Other smaller macroscopic vortex structures appear in the wake during the transient due to the variations of sign in the shed vorticity produced by the body motion (see Fig. 15, first row). Note that the wake is not flat close to the section during the early transient phase, whereas it finally becomes approximatively planar while approaching steady conditions (see Fig. 15, second row).

\subsection{Aeroelastic response to a body-vortex interaction}

The aeroelastic model is finally used to simulate the response of the flat-plate airfoil to the interaction with a passing vortex disturbing a steady-state configuration. The section properties are the same as in the preceding simulations. The angle of vanishing elastic moment is $\alpha_{e}=5^{\circ}$. In all the simulations, the vortex circulation is assumed to be equal (in modulus) to the stationary one $\tilde{\Gamma}_{b}$ around the section. Four situations are examined: the cases of a vortex passing either above or below the plate for two values of $u_{\infty}$, identifying two initial aeroelastostatic configurations given by Eq. (25). Specifically, the vortex circulation is assumed as 

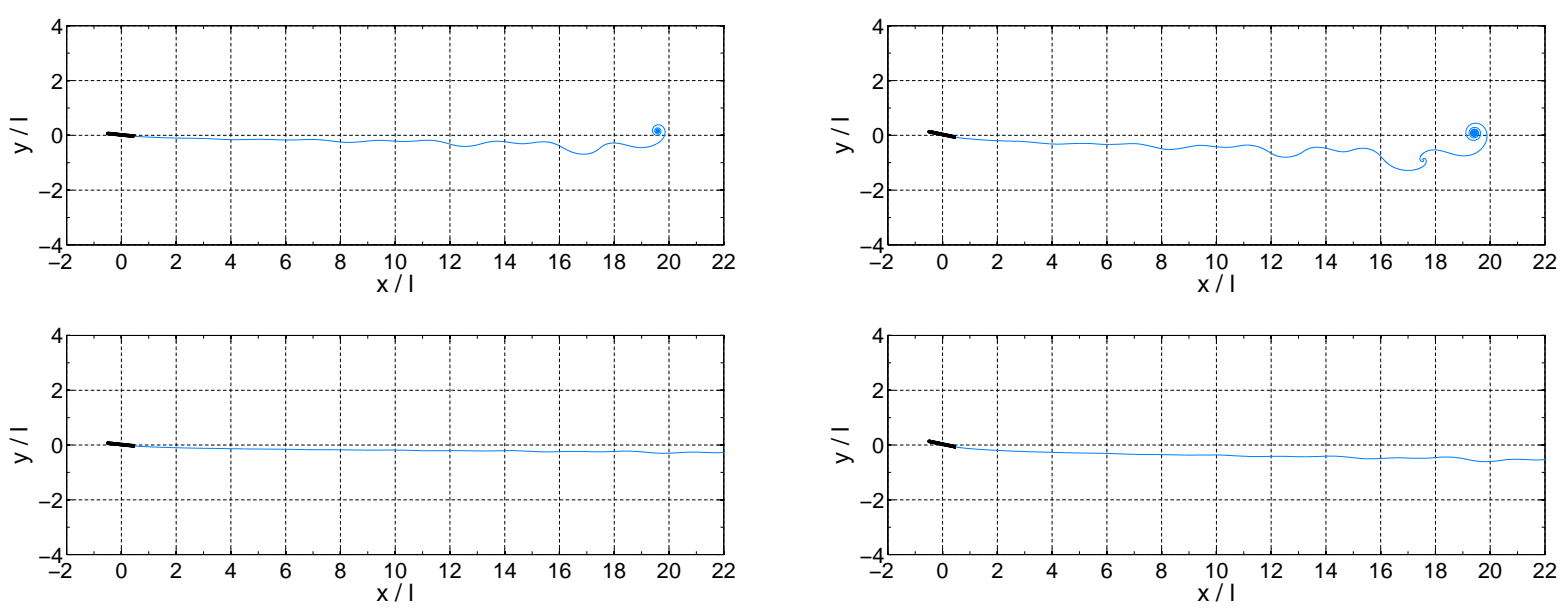

(a)

(b)

Figure 15: Sudden start: wake configurations at the non-dimensional times $2 t u_{\infty} / l=40$ (first row), 80 (second row) for $\alpha_{0}=5^{\circ}$ $(a), 10^{\circ}(b)$ and $u_{\infty}=10 \mathrm{~m} / \mathrm{s}$ (Tests 1 and 2$)$.

$\begin{array}{cccccccc}\text { Test } & u_{\infty}(\mathrm{m} / \mathrm{s}) & \tilde{H}_{y}(\mathrm{~cm}) & \tilde{\alpha}(\mathrm{deg}) & \tilde{\Gamma}_{b}\left(\mathrm{~m}^{2} / \mathrm{s}\right) & \Gamma_{v} & y_{v}(0) & x_{v}(0) \\ \mathbf{5} & 2.5 & 0.09 & 5.05 & -0.6915 & +\Gamma_{b} & +0.25 \times l & -2.5 \times l \\ \mathbf{6} & 2.5 & 0.09 & 5.05 & -0.6915 & -\Gamma_{b} & -0.25 \times l & -2.5 \times l \\ \mathbf{7} & 10 & 1.7 & 5.96 & -3.2616 & +\Gamma_{b} & +0.50 \times l & -2.5 \times l \\ \mathbf{8} & 10 & 1.7 & 5.96 & -3.2616 & -\Gamma_{b} & -0.50 \times l & -2.5 \times l\end{array}$

Table 4: Body-vortex interaction: free-stream velocity $\left(u_{\infty}\right)$, stationary configuration $\left(\tilde{H}_{y}, \tilde{\alpha}, \tilde{\Gamma}_{b}\right)$, vortex circulation $\left(\Gamma_{v}\right)$ and initial position $\left(x_{v}(0), y_{v}(0)\right)$.

$\Gamma_{v}=+\tilde{\Gamma}_{b}$ (clockwise) when the vortex initial position is above the $x$-axis, whereas it is assumed as $\Gamma_{v}=-\tilde{\Gamma}_{b}$ (counterclockwise) when the vortex is initially placed below the $x$-axis. The case studies and related equilibrium quantities are listed in Tab. 4. The numerical results are presented in terms of relative variations in the quantities of interest (vertical plate-center position, angle of attack, aerodynamic coefficients) with respect to the initial steady-state values.

Figure 16 illustrates the relative variations in the plate-center plunge and in the angle of attack for the four case studies. As the vortex approaches the section, the induced velocity field is such that pressure increases on the suction side (when the clockwise vortex passes above, in Tests 5 and 7 ) or on the pressure side (when the counterclockwise vortex passes below, in Tests 6 and 8). Therefore, the section is pushed away from the vortex in all the case studies. The body-vortex interaction excites plunge and pitch motions differently depending on the fly-over time $l / u_{\infty}$ (namely the time to travel from the leading to the trailing edge with velocity $u_{\infty}$ ). Indeed, the lower velocity results in a slower passage of the vortex in the region closer to the section, whereas a quicker passage occurs in the case of the higher velocity. Since the fly-over time is equal to $0.4 \mathrm{~s}$ for $u_{\infty}=2.5$ $\mathrm{m} / \mathrm{s}$, corresponding to the period of the vertical bending spring, the vortex passage basically perturbs plunge without causing relevant pitch in Tests 5 and 6 (note that $T_{\alpha}=1 / f_{\alpha}=0.2 \mathrm{~s}$ ). In contrast, the fly-over time is half the period of the torsional spring for $u_{\infty}=10 \mathrm{~m} / \mathrm{s}$, so that the vortex passage excites pitch and, as 

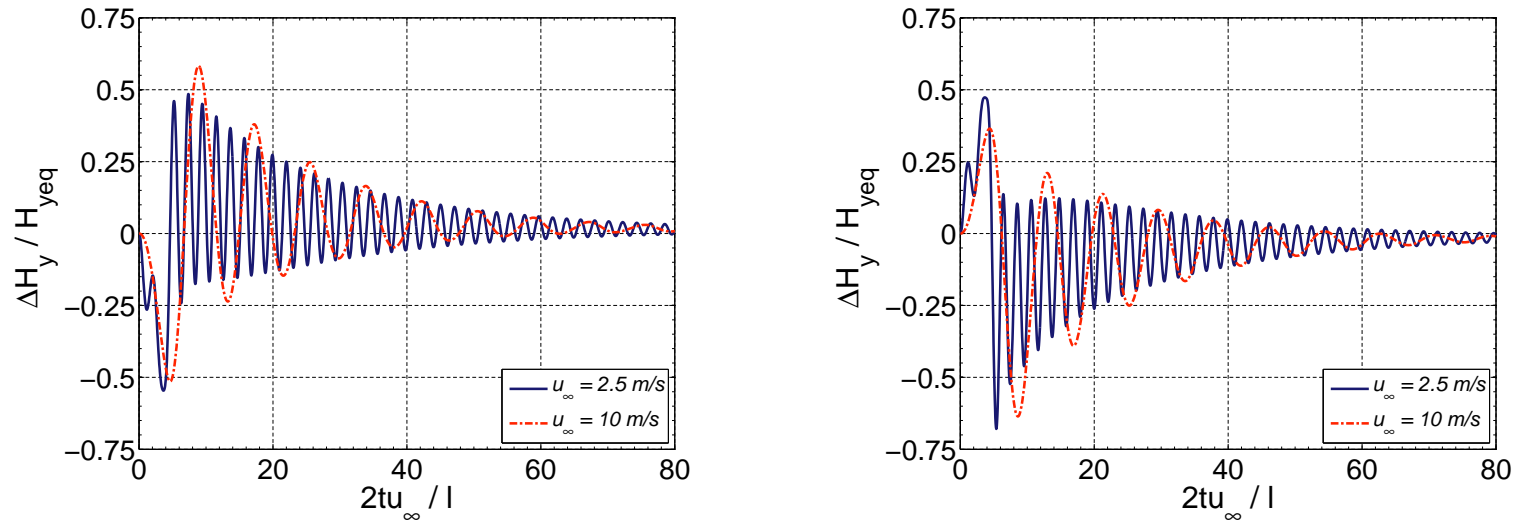

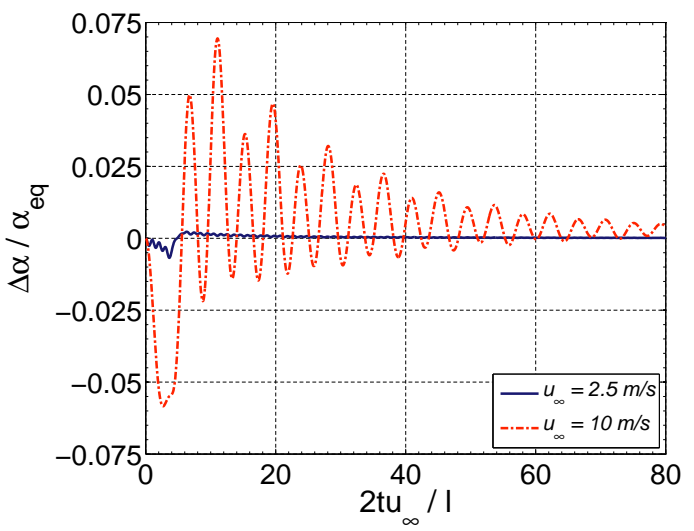

(a)

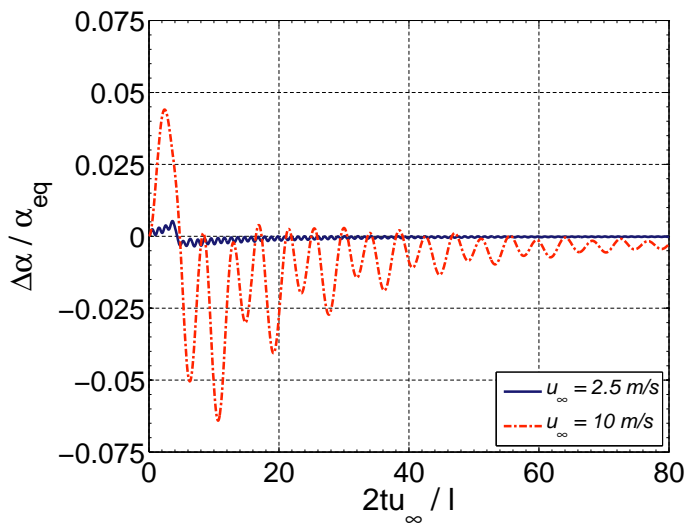

(b)

Figure 16: Body-vortex interaction: time-histories of the relative plunge at the plate center (first row) and relative angle of attack variation (second row) for the vortex passing above $(a)$ or below $(b)$ the section (Tests $5-8)$. 

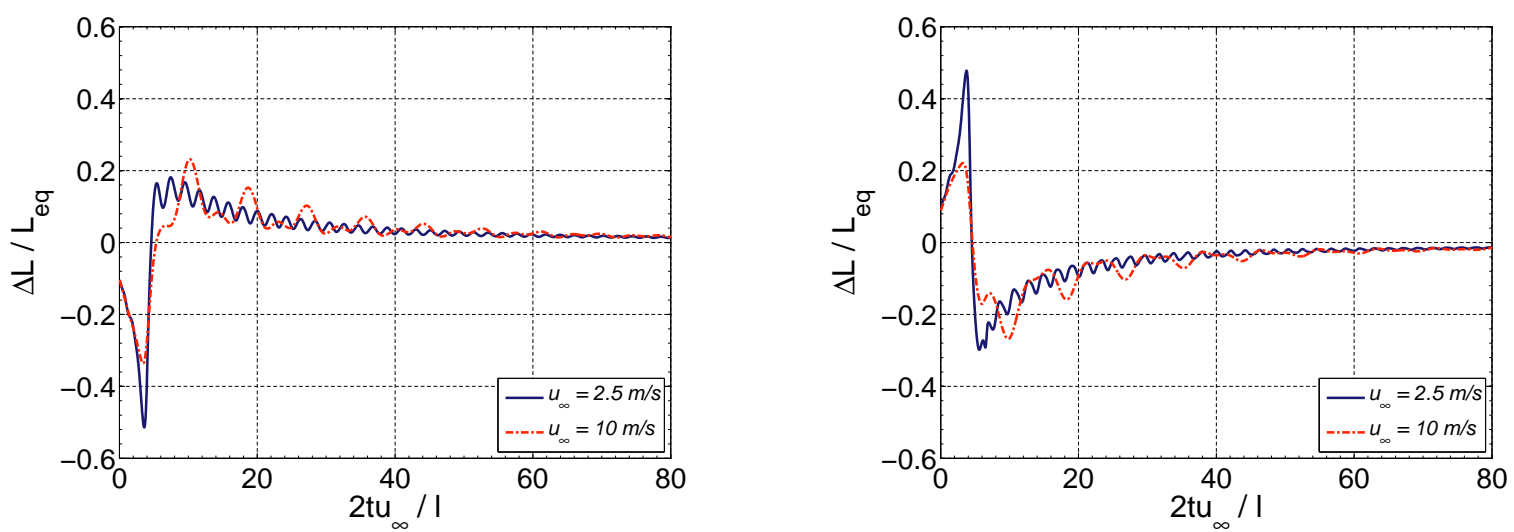

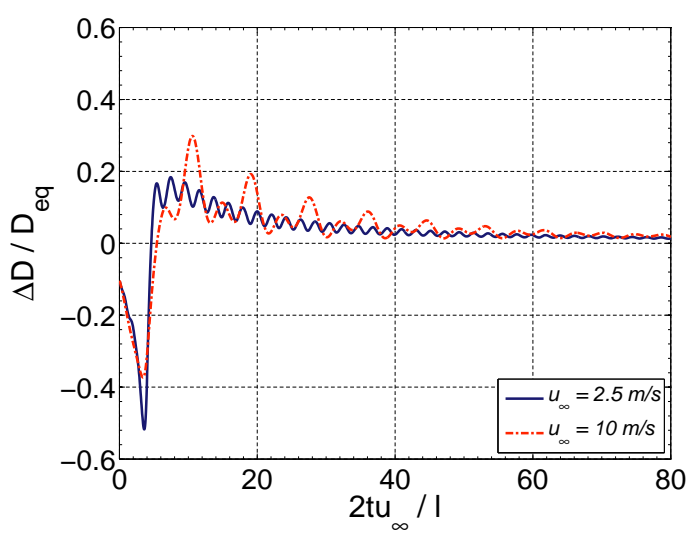

(a)

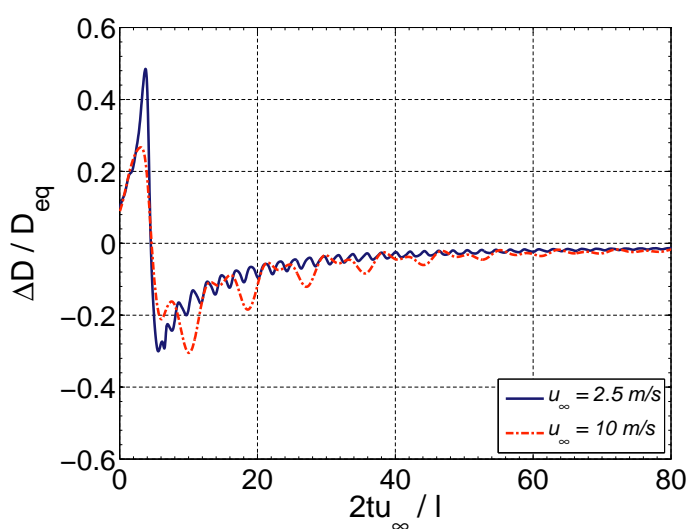

(b)

Figure 17: Body-vortex interaction: time-histories of the relative variations in the lift (first row) and drag (second row) coefficients for the vortex passing above $(a)$ or below $(b)$ the section (Tests $5-8$ ).

a consequence, plunge as well. Note that wake diffusion is not expected to significantly affect the aeroelastic response, due to the fact that the convective time $l / u_{\infty}$ is very small compared to the diffusive time $l^{2} / \nu(\nu$ is the kinematic viscosity of the fluid).

The relative variations in the lift, drag, and moment coefficients are presented in Figs. 17 and 18. The peaks occur at the same non-dimensional time, as expected, but at different physical times depending on $u_{\infty}$. The time-history of the non-dimensional location of the center of pressure along the chord is presented in Fig. 19. The center of pressure leaves the quarter-chord as the vortex passage perturbs the flow, then it recovers the initial location at the end of the transient phase.

For the sake of completeness, some wake configurations are presented in Figs. 20 and 21. Spatial coordinates are in unit chord. Figure 20 shows that an asymmetric dipole emerges as the vortex flows downstream due to its interaction with the wake vorticity. Because of the lack of symmetry, the dipole rotates clockwise if the vortex has negative circulation (Fig. $20(a)$ ) and counterclockwise in the other case (Fig. $20(b)$ ). A closer view of the dipoles for $u_{\infty}=10 \mathrm{~m} / \mathrm{s}$ is presented in Fig. 21. The wake configurations point out the importance of free-wake modeling to capture relevant features of the mutual interaction between the section, the vortex, and the wake. Indeed, a free-wake approach can account for relevant phenomena such as the deviation of the vortex path close 


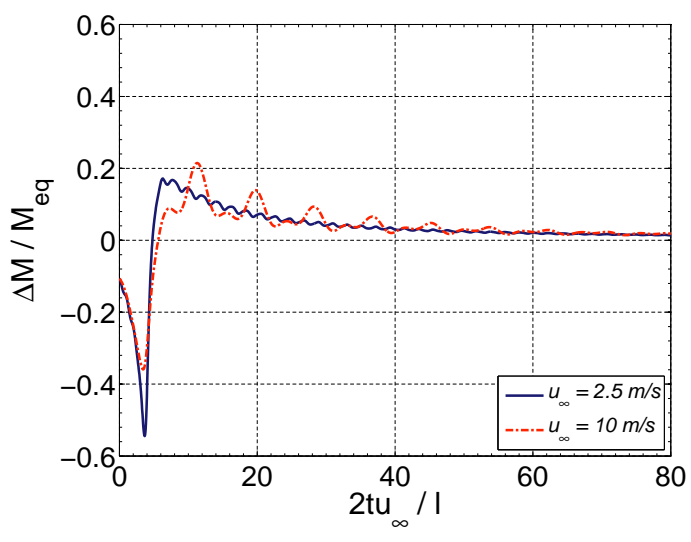

(a)

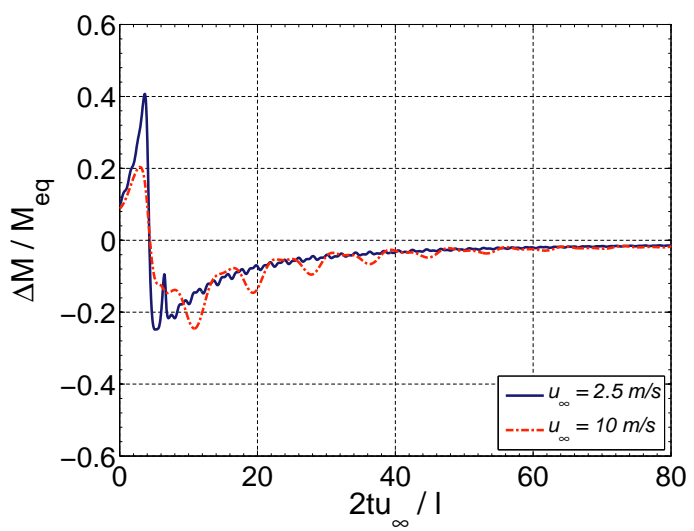

(b)

Figure 18: Body-vortex interaction: time-histories of the relative variation in the pitching moment coefficient for the vortex passing above $(a)$ or below $(b)$ the section (Tests $5-8$ ).

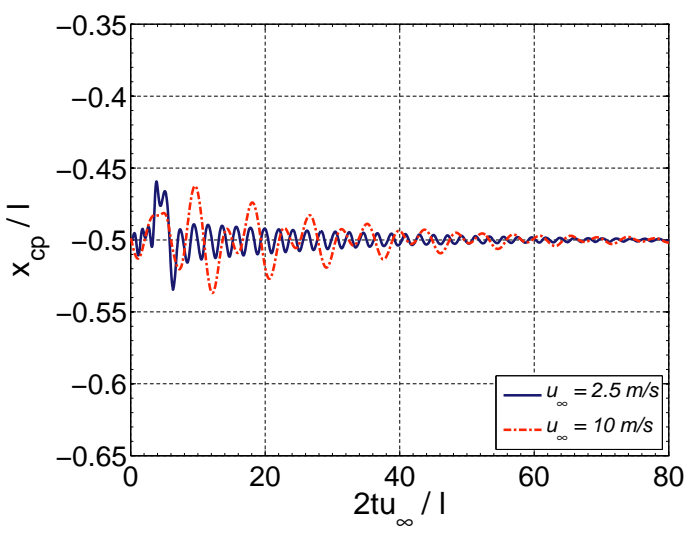

(a)

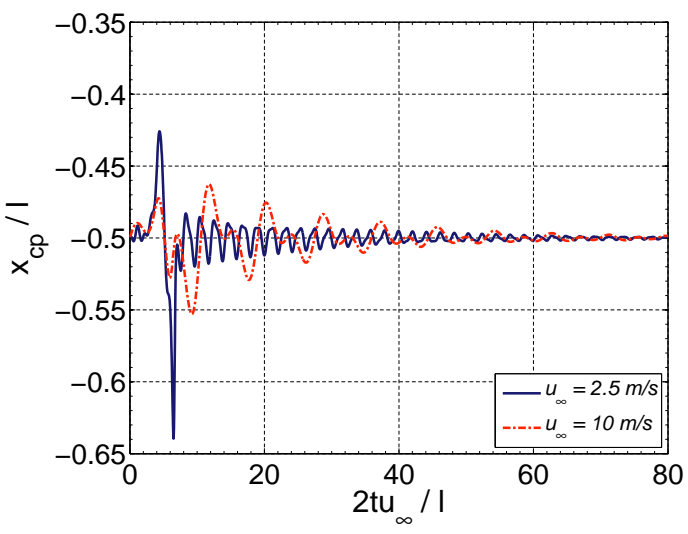

(b)

Figure 19: Body-vortex interaction: time-histories of the non-dimensional location of the center of pressure along the chord for the vortex passing above $(a)$ or below $(b)$ the section (Tests $5-8$ ).
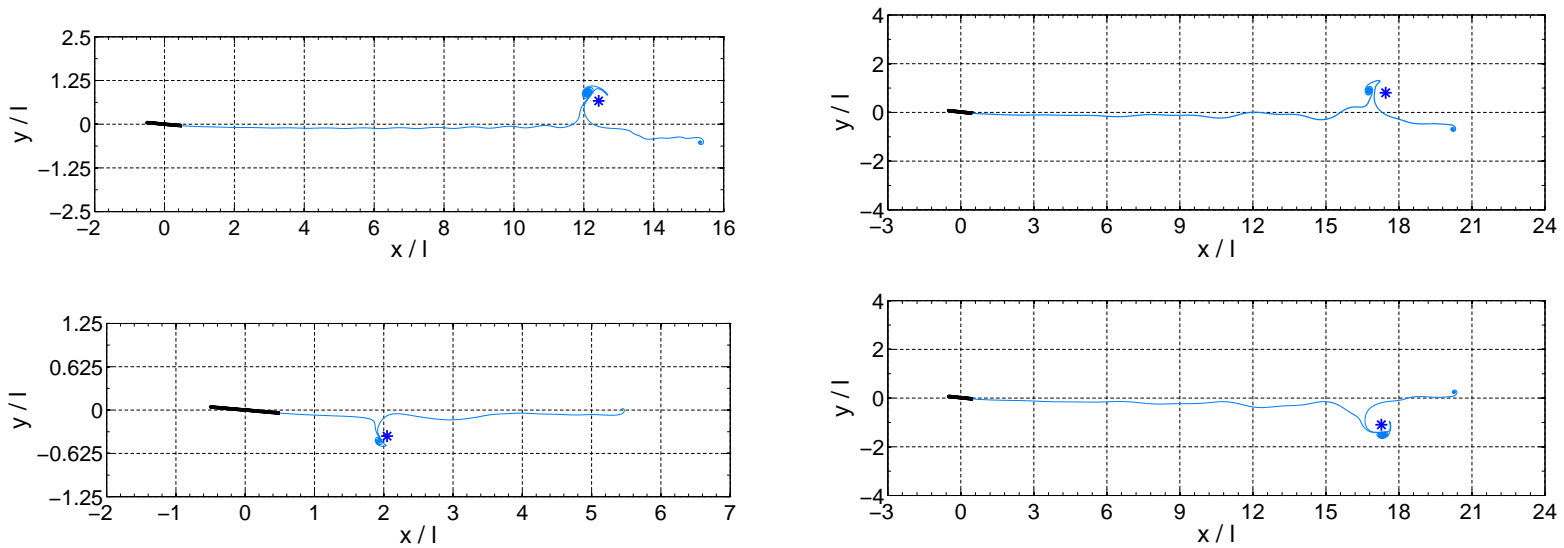

(a)

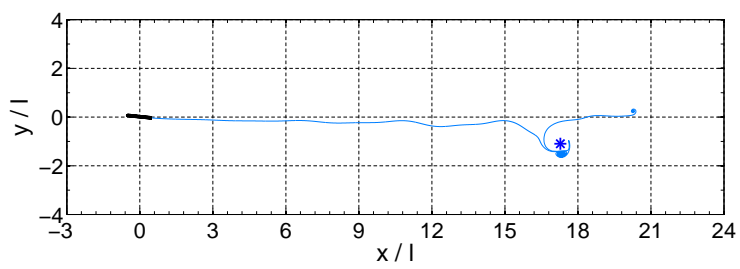

(b)

Figure 20: Body-vortex interaction: wake configurations for $u_{\infty}=2.5 \mathrm{~m} / \mathrm{s}(a)$ at the non-dimensional time $2 t u_{\infty} / l=30$ (first row) and $2 t u_{\infty} / l=10$ (second row) and for $u_{\infty}=10 \mathrm{~m} / \mathrm{s}(b)$ at the non-dimensional time $2 t u_{\infty} / l=40$ for the vortex passing above (first row) or below (second row) the section (Tests 5-8). 


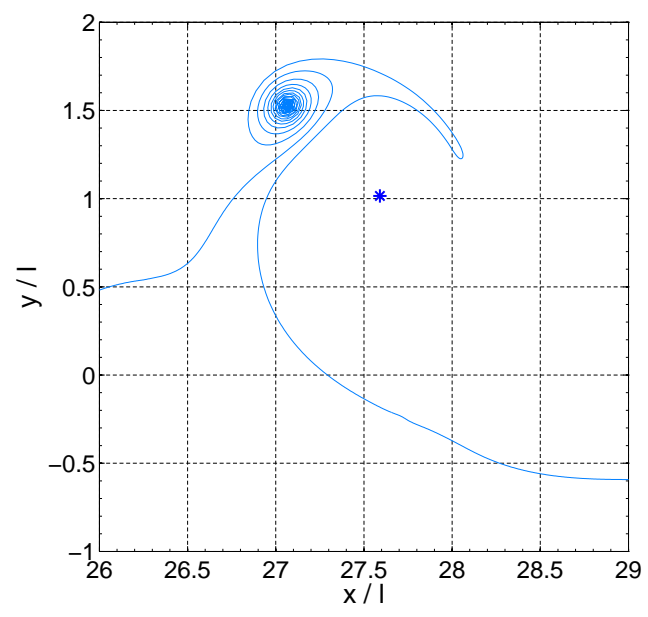

(a)

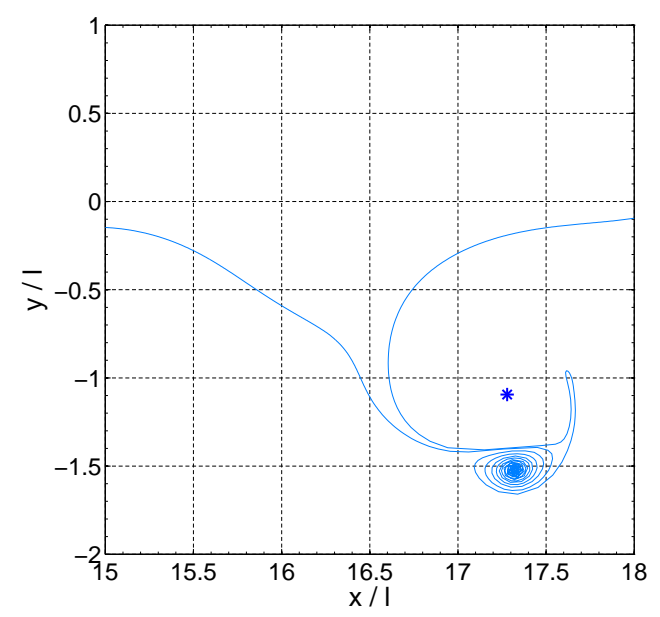

(b)

Figure 21: Body-vortex interaction: dipoles at the non-dimensional time $2 t u_{\infty} / l=60$ for the vortex passing above the section $(a)$ and at the non-dimensional time $2 t u_{\infty} / l=40$ for the vortex passing below the section $(b)$ for $u_{\infty}=10 \mathrm{~m} / \mathrm{s}($ Tests 7 and 8 ).

to the plate and the generation of dipoles, leading to an accurate prediction of the aerodynamic loads on the section and, consequently, of the aeroelastic response.

\section{Concluding remarks}

A nonlinear modeling based on conformal mapping is presented to obtain semi-analytical formulas for the unsteady aerodynamic force and pitching moment on moving airfoils in incompressible potential flow. The aerodynamic loads have been derived by locally imposing the no-penetration unsteady boundary condition on the body wall, with no theoretical restriction on the section shape and motion. In order to assess the fidelity of the modeling approach, the general formulation has been specialized to the case of a flat-plate airfoil in arbitrary plunge, surge, and pitch, with the aim to address a flexible thin airfoil in future research. The unsteady aerodynamic model features a complex-potential representation of the flow and a vortex method to include free-wake dynamics. The aerodynamic loads on the flat-plate airfoil have been evaluated via the residue theorem. Semi-analytical formulas for the aerodynamic force and pitching moment have been presented and used to develop a free-wake aeroelastic model for a flat-plate airfoil elastically connected to a support.

The unsteady aerodynamic model has been validated with classical linearized solutions as well as with experimental data and theoretical predictions for a large-amplitude pitch-up, hold, pitch-down maneuver. The aeroelastic model has been then used to study the response of a flat-plate airfoil to sudden starts and body-vortex interactions. The influence of assuming flat wake in place of free-wake dynamics has been critically discussed. Concerning aeroelastic response to sudden starts, free-wake effects have been shown to be more relevant for higher-amplitude pitch motions, due to the fact that the flat-wake assumption becomes less representative of the actual wake dynamics. The need of free-wake modeling in order to explore body-vortex interactions has been also proved. Indeed, relevant phenomena such as the deviation of the vortex path close to the section or the generation of dipoles could not be captured by a flat-wake aerodynamic model. 
The proposed modeling approach has demonstrated a higher predictive capability with respect to linearized formulations, and could be used to effectively model the aeroelastic behavior of airfoils undergoing arbitrary motion in a time-dependent potential stream of incompressible fluid. Future research will address the development of a semi-analytical, free-wake unsteady aerodynamic model for thin airfoils featuring chordwise deformation, with the aim to point out flexibility effects on aeroelastic stability and response as well as to explore suitable combinations of rigid-body and elastic motion to enhance thrust generation.

\section{Acknowledgments}

This paper has been supported by the Finanziamento Progetti di Ricerca Anno 2014, University of Rome La Sapienza. The authors wish to acknowledge the support of Prof. Ashok Gopalarathnam, Dr. Kiran Ramesh, and Dr. Zhimiao Yan in proving experimental and numerical data for validating the present unsteady aerodynamic model. The authors would also like to thank Luigi Morino for his important contribution in reviewing the paper and commenting the results.

\section{A. On the desingularization of the Biot-Savart kernel}

In this appendix, the use of the smoothed Biot-Savart kernel given by Eq. (19) is briefly discussed. This corresponds to consider the desingularized Green function:

$$
G_{\varepsilon}(\boldsymbol{x})=\frac{1}{4 \pi} \log \left(|\boldsymbol{x}|^{2}+\varepsilon^{2}\right)
$$

satisfying the desingularized equation:

$$
\nabla^{2} G_{\varepsilon}(\boldsymbol{x})=\frac{1}{\pi} \frac{\varepsilon^{2}}{\left(|\boldsymbol{x}|^{2}+\varepsilon^{2}\right)^{2}}:=F_{\varepsilon}(\boldsymbol{x}),
$$

whereas the Green function $G$ satisfies the well-known equation involving the Dirac delta function $\nabla^{2} G(\boldsymbol{x})=$ $\delta(\boldsymbol{x})$. Note that $F_{\varepsilon} \rightarrow \delta$ in the limit as $\varepsilon \rightarrow 0$.

The Fourier transform ${ }^{3}$ of the Green function $G$ is given by $\boldsymbol{g}(\boldsymbol{k})=-1 /(2 \pi|\boldsymbol{k}|)^{2}$, so that using this result and Eq. (30) yields:

$$
\boldsymbol{g}_{\varepsilon}=(2 \pi)^{2} \boldsymbol{g} \boldsymbol{f}_{\varepsilon}=\mathcal{F}\left[G \star F_{\varepsilon}\right],
$$

where $\boldsymbol{g}_{\varepsilon}$ and $\boldsymbol{f}_{\varepsilon}$ denote the Fourier transforms of $G_{\varepsilon}$ and $F_{\varepsilon}$, respectively. Therefore, the desingularized Green function in Eq. (29) is also obtained as the convolution $G \star F_{\varepsilon}$.

This last result provides a simple interpretation of the desingularization procedure adopted. Indeed, using the smoothed Biot-Savart kernel given by Eq. (19) is equivalent to assume the vorticity field $\left(\omega \star F_{\varepsilon}\right)(\boldsymbol{x})$ in place

\footnotetext{
${ }^{3}$ The Fourier transform $\boldsymbol{p}(\boldsymbol{k})$ of the function $P(\boldsymbol{x})$ is evaluated as:

$$
\boldsymbol{p}(\boldsymbol{k})=\mathcal{F}[P](\boldsymbol{k})=\frac{1}{(2 \pi)^{2}} \int_{R_{2}} d A(\boldsymbol{x}) \exp (-\boldsymbol{i k} \cdot \boldsymbol{x}) P(\boldsymbol{x}) .
$$
}




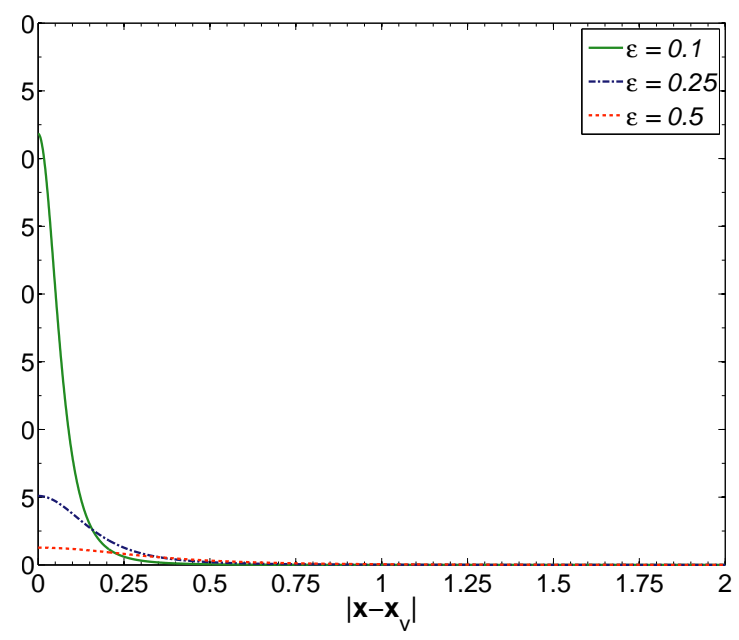

Figure 22: Function $F_{\varepsilon}$ versus $\left|\boldsymbol{x}-\boldsymbol{x}_{v}\right|$ for three values of the smoothing parameter $\varepsilon=0.1,0.25,0.5$.

of the original field $\omega(\boldsymbol{x})=\Gamma_{v} \delta\left(\boldsymbol{x}-\boldsymbol{x}_{v}\right)$ given by the point-vortex approximation for a single point vortex of circulation $\Gamma_{v}$ placed at $\boldsymbol{x}=\boldsymbol{x}_{v}$. The desingularized vorticity field is given by:

$$
\omega \star F_{\varepsilon}(\boldsymbol{x})=\int_{R_{2}} d A(\boldsymbol{y}) \omega(\boldsymbol{y}) F_{\varepsilon}(\boldsymbol{x}-\boldsymbol{y})=\Gamma_{v} F_{\varepsilon}\left(\boldsymbol{x}-\boldsymbol{x}_{v}\right) .
$$

In Fig. 22 the function $F_{\varepsilon}$ is plotted versus $\left|\boldsymbol{x}-\boldsymbol{x}_{v}\right|$ for three values of $\varepsilon$. The figure shows that the circulation originally concentrated at the vortex location in the case of the vorticity field $\omega$ is spread in the surrounding region when applying the desingularization procedure. The spread around the vortex location increases with the smoothing parameter.

\section{B. Derivation of the Theodorsen solution}

The computations for recovering the Theodorsen solution in Eq. (26) from the present linearized formulas in Eq. (27) for the lift and pitching moment are here presented.

First of all, note that the non-circulatory part of the aerodynamic loads in Eq. (27) is identical to the one in Eq. (26). To recast the circulatory part in terms of vortex positions and velocities in the physical plane, the quantities $a_{x}^{(1)}, \dot{a}_{x}^{(1)}$, and $\dot{a}_{x}^{(2)}$ are rewritten by means of Eq. (28) as follows $(R=l / 4)$ :

$$
\begin{aligned}
& a_{x}^{(1)}=\quad+\frac{l}{4} \sum_{j=1}^{n} \frac{\Gamma_{j}}{\zeta_{x j}}=+\frac{2}{l} \sum_{j=1}^{n} \Gamma_{j}\left(\xi_{j}-\sqrt{\xi_{j}^{2}-l^{2} / 4}\right), \\
& \dot{a}_{x}^{(1)}=-\frac{l}{4} \sum_{j=1}^{n} \frac{\Gamma_{j}}{\zeta_{x j}^{2}} \dot{\zeta}_{x j}=-\frac{2}{l} u_{\infty} \sum_{j=1}^{n} \frac{\Gamma_{j} \xi_{j}}{\sqrt{\xi_{j}^{2}-l^{2} / 4}}-\frac{2}{l} u_{\infty} \Gamma_{b}, \\
& \dot{a}_{x}^{(2)}=-\frac{l^{2}}{8} \sum_{j=1}^{n} \frac{\Gamma_{j}}{\zeta_{x j}^{3}} \dot{\zeta}_{x j}=+\frac{16}{l^{2}} u_{\infty} \sum_{j=1}^{n} \Gamma_{j} \frac{\xi_{j} \sqrt{\xi_{j}^{2}-l^{2} / 4}-\xi_{j}^{2}+l^{2} / 8}{\sqrt{\xi_{j}^{2}-l^{2} / 4}},
\end{aligned}
$$


having used Kelvin theorem $\left(\Gamma_{b 0}=0\right)$. Therefore, the circulatory terms in Eq. (27) become:

$$
\begin{aligned}
-\rho u_{\infty} \Gamma_{b}-\rho \frac{l}{2} \dot{a}_{x}^{(1)} & =\rho u_{\infty} \sum_{j=1}^{n} \frac{\Gamma_{j} \xi_{j}}{\sqrt{\xi^{2}-l^{2} / 4}}, \\
+\rho u_{\infty} \frac{l}{2} a_{x}^{(1)}-\rho \frac{l^{2}}{16} \dot{a}_{x}^{(2)} & =\rho u_{\infty} \frac{l^{2}}{8} \sum_{j=1}^{n} \frac{\Gamma_{j}}{\sqrt{\xi^{2}-l^{2} / 4}},
\end{aligned}
$$

which correspond to those by Theodorsen for the case of $n$ vortices.

\section{References}

[1] Wagner, H., "Über die Entstehung des Dynamischen Auftriebes von Tragflügeln", ZAMM, Vol. 5, No. 1, 1925, pp. 17-35.

[2] Küssner, H. G., "Zusammenfassender Bericht über den instationren Auftrieb von Flügeln", Luftfahrtforschung, Vol. 13, No. 12, 1936, pp. 410-424.

[3] Theodorsen, T., General Theory of Aerodynamic Instability and the Mechanism of Flutter, NACA TR No. 496, 1935.

[4] Bisplinghoff, R. L., Ashley, H., and Halfman, R. L., Aeroelasticity, Dover Publications, Mineola, NY, 1996.

[5] Theodorsen, T., and Garrick, I. E., "Mechanism of Flutter. A Theoretical and Numerical Investigation of the Flutter Problem", NACA TR No. 685, 1940.

[6] Garrick, I. E., "On Some Reciprocal Relations in the Theory of Nonstationary Flows", NACA TR No. 629, 1938.

[7] Isaacs, R., "Airfoil Theory for Rotary Wing Aircraft", Journal of the Aeronautical Sciences, Vol. 12, No. 1, 1945, pp. 113-117.

[8] Greenberg, J. M., "Airfoil in Sinusoidal Motion in a Pulsating Stream", NACA TN No. 1326, 1947.

[9] Edwards, J. W., Ashley, and H., Breakwell, J. V., "Unsteady Aerodynamic Modeling for Arbitrary Motions", AIAA Journal, Vol. 17, No. 4, 1979, pp. 365-374.

[10] Edwards, J. H., "Application of Laplace Transform Methods to Airfoil Motion and Stability Calculations", AIAA Paper 79-0772, 1979.

[11] Jones, R. T., "Operational Treatment of the Nonuniform Lift Theory to Airplane Aerodynamics", NACA Rep. 667, 1938.

[12] Jones, R. T., "The Unsteady Lift of a Wing of Finite Aspect Ratio", NACA Rep. 681, 1938.

[13] Venkatesan, C., and Friedmann, P., P., "New Approach to Finite-State Modeling of Unsteady Aerodynamics", AIAA Journal, Vol. 24, No. 12, 1986, pp. 1889-1897. 
[14] Peters, D. A., Karunamoorthy, S., and Cao, W., "Finite State Induced Flow Models. Part I: TwoDimensional Thin Airfoil", Journal of Aircraft, Vol. 32, No. 2, 1995, pp. 313-322.

[15] Peters, D. A., "Two-dimensional Unsteady Airfoil Theory. An Overview.", Journal of Fluids and Structures, Vol. 24, No. 3, 2008, pp. 295-312.

[16] Ramesh, K., Gopalarathnam, A., Edwards, J. R., Ol, M. V., and Granlund, K., "An unsteady airfoil theory applied to pitching motions validated against experiment and computation", Theoretical Computational Fluid Dynamics, Vol. 27, 2013, pp. 843-864.

[17] Ramesh, K., Gopalarathnam, A.,Granlund, K., Ol, M. V., and Edwards, J. R., "Discrete-vortex method with novel shedding criterion for unsteady aerofoil flows with intermittent leading-edge vortex shedding", Journal of Fluid Mechanics, Vol. 751, 2014, pp. 500-538.

[18] Xia, X., Mohseni, K., "Lift Evaluation of a Two-dimensional Pitching Flat Plate", Physics of Fluids, Vol. 25, No. 091901, 2013, pp. 1-26.

[19] Sarpkaya, T., "An Inviscid Model of Two-Dimensional Vortex Shedding for Transient and Asymptotically Steady Separated Flow Over an Inclined Flat Plate", Journal of Fluid Mechanics, Vol. 68, No. 1, 1975, pp. 109-128.

[20] Kiya, M., and Arie, M., "A Contribution to an Inviscid Vortex-Shedding Model for an Inclined Flat Plate in Uniform Flow", Journal of Fluid Mechanics, Vol. 82, No. 2, 1977, pp. 223-240.

[21] Batchelor, G. K., An Introduction to Fluid Dynamics, Cambridge University Press, Cambridge, England, UK, 1967.

[22] Milne-Thomson, L. M., Theoretical Hydrodynamics, Dover Publications, Mineola, NY, 1996.

[23] Ablowitz, M. J., and Fokas, A. S., Complex Variables: Introduction and Applications, $2^{\text {nd }}$ ed., Cambridge University Press, Cambridge, England, UK, 2003.

[24] Wang, C., and Eldredge, J. D., "Low-order Phenomenological Modeling of Leading-Edge Vortex Formation", Theoretical Computational Fluid Dynamics, Vol. 27, 2013, pp. 577-598.

[25] Yan, Z., Taha, H. E., and Hajj, M. R., "Geometrically-exact Unsteady Model for Airfoils Undergoing Large", Aerospace Science and Technology, Vol. 39, 2014, pp. 293-306.

[26] Walker, W. P., and Patil, M. J., "Unsteady Aerodynamics of Deformable Thin Airfoils", Journal of Aircraft, Vol. 52, No. 6, 2014, pp. 1673-1680.

[27] Davis, P. J., The Schwarz Function and its Applications, Carus Mathematical Monographs, Mathematical Association of America, Buffalo, NY, 1974.

[28] Riccardi, G., and Durante, D., Elementi di Fluidodinamica, Springer Verlag, 2006. 
[29] Majda, A. J., and Bertozzi, A. L., Vorticity and Incompressible Flow, Cambridge University Press, Cambridge, England, UK, 2002 .

[30] Krasny, R. "Desingularization of Periodic Vortex Sheet Roll-up", Journal of Computational Physics, Vol. 65, 1986, pp. 292-313.

[31] Peters, D. A., and Hsieh, M. A., "A State-space Airloads Theory for Flexible Airfoils", Journal of American Helicopter Society, Vol. 52, No. 4, 2007, pp. 329-342.

[32] Ol, M. V., Altman, A., Eldredge, J. D., Garmann, D. J., and Lian, Y., "Rsum of the AIAA FDTC Low Reynolds Number Discussion Group's Canonical Cases", AIAA paper 2010-1085, 2010. 